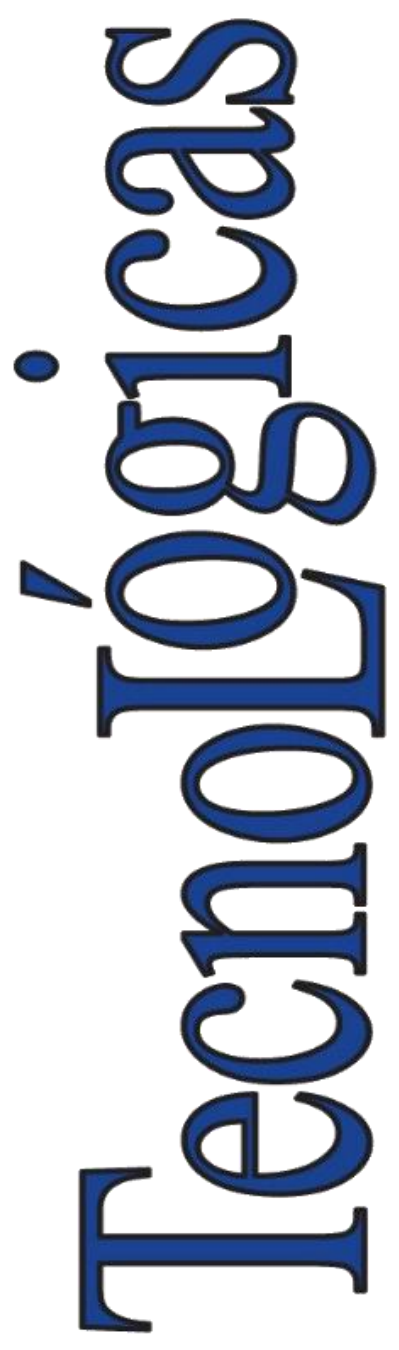

ISSN-p: 0123-7799

ISSN-e: $2256-5337$

Vol. 24, nro. 51, e1880, 2021

Recibido: 22 enero 2021 Aceptado: 25 mayo 2021 Disponible: 9 junio 2021

CInstituto Tecnológico Metropolitano

Este trabajo está licenciado bajo una Licencia Internacional

Creative Commons Atribución (CC BY-NC-SA)

\section{Sistema de gestión de energía descentralizado basado en multiagentes para operación de múltiples microrredes}

\section{Decentralized Energy Management System Based on Multi-agents to Operate Multiple Microgrids}

iD Alfredo Sánchez-Silvera ${ }^{1}$;

(iD) José Guillermo Guarnizo-Marín²;

(iD)

Edwin Francisco Forero-García ${ }^{3}$; iD Davis Montenegro-Martínez ${ }^{4}$

1 Universidad Santo Tomás, Bogotá-Colombia, alfredosanchez@usantotomas.edu.co

2 Universidad Santo Tomás, Bogotá-Colombia, jose.guarnizo@usantotomas.edu.co

${ }^{3}$ Universidad Santo Tomás, Bogotá-Colombia, edwinforero@usantotomas.edu.co

${ }_{4}^{4}$ Electric Power Research Institute, Palo Alto, California-Estados Unidos, dmmartinez@epri.com
Cómo citar / How to cite

A. Sánchez-Silvera; J. G. Guarnizo-Marín; E. F. Forero-García; D. Montenegro-Martínez, "Sistema de gestión de energía descentralizado basado en multiagentes para operación de múltiples microrredes", TecnoLógicas, vol. 24, nro. 51, e1880, 2021. https://doi.org/10.22430/22565337.1880 


\section{Resumen}

En años recientes, las microrredes han logrado un considerable desarrollo debido a que representan una alternativa técnica para responder a contingencias en la red de distribución, como también a incrementar el nivel de generación distribuida, entre otros beneficios. Por tal motivo, el presente artículo presenta un modelo de gestión energética basado en sistemas multiagentes para microrredes que operan en modo isla. El objetivo de esta investigación es el diseño de un sistema multiagente que permita gestionar el funcionamiento de un conjunto de microrredes distribuidas en un entorno aislado, además de mantener la correcta conexión con el bus común que une todas las microrredes, el sistema multiagente debe evitar sobrecargas y descargas profundas en las baterías. La metodología implementada es de tipo empírico analítica, la simulación comienza con una revisión del estado del arte, en búsqueda de una estrategia que permita coordinar un sistema de microrredes compuesto, donde estas están conectadas al mismo sistema de distribución operando en modo isla. La simulación del sistema se realizó mediante OpenDSS-G y Python. Los resultados obtenidos sugieren que un sistema de gestión de energía descentralizado, basado en la teoría de sistemas de agentes múltiples, puede tener importantes beneficios como, por ejemplo, el carácter autónomo de las microrredes para la generación de energía en zonas no interconectadas. Finalmente, con la teoría de multiagente se pueden crear sistemas de generación distribuida más confiables debido a su capacidad autónoma de toma de decisiones, para cubrir demandas eléctricas desde microrredes vecinas y conjuntamente prevenir sobrecargas y profundas descargas en las baterías.

\section{Palabras clave}

Microrredes, sistemas multiagente, gestión de energía eléctrica, sistema distribuido, Simulador OpenDSS-G.

\section{Abstract}

Microgrids have experienced a significant development in recent years because they represent a technical alternative to respond to contingencies in electrical distribution networks and increase the level of distributed generation, among other benefits. The objective of this study is to design an architecture based on multi-agent systems that can be used to manage the operating mode of a distributed microgrid system in an islanded environment. In such architecture, the correct connection of the common bus that links all the microgrids with the multi-agent system is maintained, and overloads and deep discharges in the batteries are avoided. The methodology implemented here is empirical-analytical. The simulation is based on a review of the state of the art that was conducted to find a strategy that can coordinate a composite microgrid system where the microgrids are connected to the same distribution system operating in islanded mode. The system was simulated using OpenDSS-G and Python. The results obtained suggest that a decentralized energy management system based on the theory of multi-agent systems can have important benefits; for example, the autonomous nature of microgrids for power generation in non-interconnected areas. Finally, multi-agent theory can be employed to create more reliable distributed generation systems (due to their autonomous decision-making capacity), meet the electrical demands of neighboring microgrids, and jointly prevent overcharges and deep discharges in batteries.

\section{Keywords}

Microgrids; multi-agent systems; electrical energy management; distributed system; OpenDSS-G simulation. 


\section{INTRODUCCIÓN}

La población mundial ha reconocido los inconvenientes del calentamiento global, por lo cual las fuentes de energía renovable se plantean como una solución prometedora para disminuir las emisiones de efecto invernadero. El uso de fuentes de energía renovable presenta un enorme potencial para diversas aplicaciones, especialmente para los sistemas autónomos sin conexión a la red eléctrica que muestran grandes beneficios, como es el caso de uso para las zonas no interconectadas (ZNI). En [1], los autores presentan una metodología de diseño de microrredes para ZNI que favorece la generación eléctrica para estas regiones de Colombia. Otro caso de uso se presenta en [2], donde los autores estudian el uso de fuentes renovables y la integración de recursos energéticos distribuidos (DER por su sigla en inglés) en microrredes aisladas para ZNI. Sin embargo, alimentar zonas remotas con fuentes renovables implica el uso de dispositivos de almacenamiento debido a la naturaleza fluctuante de la producción de energía [3]. Colombia no ha permanecido ajena al desarrollo de las microrredes eléctricas: en el Consejo Internacional de Grandes Sistemas Eléctricos (CIGRE), Colombia presentó un reporte técnico donde se describe el control jerárquico, la infraestructura física, tecnologías y normativas requeridas para desarrollar y promover la investigación de las microrredes eléctricas en corriente alterna (AC por sus siglas en inglés) [4].

Las microrredes son pequeñas redes de usuarios con suministro de energía local [5]. Estas redes están igualmente conectadas a una red de distribución; sin embargo, las microrredes tienen la capacidad de operar de forma independiente o de modo aislado, separándose de la red de distribución. Para suplir la demanda local de energía, las microrredes representan una alternativa técnica para responder a contingencias en la red de distribución [6] y mejorar su sistema [7], entre otros aportes. Estas cuentan con sistemas de generación y almacenamiento, como los mencionados anteriormente.

Las microrredes eléctricas han logrado un considerable desarrollo a través de las fuentes renovables de energía (RES por su sigla en inglés). En este escenario, las microrredes eléctricas se presentan apropiadas para la incorporación de las fuentes renovables, ya que estas logran funcionar aisladas de la red eléctrica o interconectadas a esta misma [8]. Cuando la microrred se encuentra interconectada, la red eléctrica establece el voltaje y la frecuencia en el bus de interconexión, al mismo tiempo los recursos energéticos distribuidos DER logran adquirir y suministrar energía en la red [9]. Debido a esto, la red eléctrica mantiene la relación entre la potencia generada y el consumo energético [10].

En el caso de operación en modo aislado, es necesario utilizar dispositivos de almacenamiento de energía (ESS por su sigla en inglés), los cuales permiten mantener un equilibrio en la potencia de generación y de consumo, así como modificar la inestabilidad inesperada de las RES. Para esto es necesario mantener la potencia, lo cual se hace muy complicado en las microrredes aisladas, ya que uno de los dispositivos DER (RES-ESS) tiene que adquirir el comportamiento de formador de red y asumir la responsabilidad de establecer la frecuencia y el voltaje de la red formada [11]. Para cumplir con los objetivos de mantener frecuencia y voltaje se desarrollan las siguientes dos estrategias: la primera es configurar un dispositivo como formador de red; y la segunda es por multimaestro, es decir, dos o más dispositivos funcionando en paralelo como formador de red [9]. En la estrategia multimaestro, la potencia es repartida en los diferentes sistemas de almacenamiento de energía. Estos sistemas conformados se pueden configurar como formador de red [12]. Para el funcionamiento adecuado de una microrred en modo isla, es fundamental precisar una arquitectura de verificación para coordinar el funcionamiento entre las diferentes DER. Esta arquitectura debe permitir la verificación de la potencia disponible en las RES, la capacidad 
de almacenamiento de energía de los ESS y la demanda de la carga [13]. Es de tener en cuenta que el estado de la carga (SOC por su sigla en inglés) de un ESS es posiblemente la variable primordial en una arquitectura de control. Esto es importante ya que puede prevenir el exceso en los límites de carga y descargas de las ESS, permitiendo ampliar su vida útil, diseñados a partir de baterías [14].

Con el avance de los sistemas multiagente, nuevos dispositivos se han desarrollado hasta el punto de ser utilizados en los sistemas eléctricos de potencia [15]; en la literatura se evidencian estas investigaciones y la aplicación de sistemas multiagente para el funcionamiento de una microrred. Se puede encontrar el diseño de estrategias donde utilizan teoría de juegos para incorporar la energía generada por redes eléctricas inteligentes (REI) [16] con el objetivo de integrar y solucionar el conflicto presentado entre agentes generadores, cliente y la red de agentes. En [17] es presentada la arquitectura de un sistema multiagente que permite la predicción y gestión en REI y, adaptadas para centrales eléctricas virtuales, los agentes están diseñados a partir de redes neuronales que pueden pronosticar la demanda energética para clientes hogar. En [18] es mostrado un algoritmo distribuido que utiliza agentes inteligentes que restituyen el servicio mediante acumuladores de energía, localización y aislamiento de fallos. En [19] se observa cómo los sistemas inmunes son usados como agentes inteligentes en el diseño de algoritmos de optimización para la gestión de DER.

Microrredes inteligentes son organizadas como agentes en [20], con el objetivo de cubrir la demanda interna e intercambiar energía con la red eléctrica principal y con otras microrredes, utilizando una estrategia de control descentralizada. En [21] se presenta una arquitectura de control descentralizada para microrredes junto con un entorno de toma de decisiones en tiempo real, basada en agentes capaces de operar en modo interconectado a la red o en isla.

Los principios de diseño y el estándar para diseño de microrredes son expuestos en [22] donde es aplicado el control jerárquico y el almacenamiento de energía en microrredes.

Puntualmente, la teoría multiagente es aplicada, según los artículos encontrados, en el control para una microrred con el propósito de gestionar su operación de conexión a la red eléctrica u operación en modo aislado. En [23] se plantea una estrategia de control de frecuencia apoyada en un sistema de múltiples agentes para una microrred autónoma, utilizando algoritmo de consenso. En [24] se propone una estrategia de control secundaria basada en multiagentes para una microrred en modo isla; el control está descentralizado y se emplea una estructura de control de dos capas sin un controlador central para microrred. Un control híbrido-jerárquico basado en múltiples agentes para una microrred inteligente es propuesto en [25] con el fin de mantener un adecuado voltaje y lograr provechos ambientales y económicos favorables. En [26] se plantea un sistema multiagente desde arquitecturas trabajadas en los robots de fútbol; los conceptos de percepción global y control centralizado son utilizados para la supervisión de una microrred eléctrica en isla; no obstante, el trabajo no contempla la coordinación de múltiples microrredes interconectadas entre sí.

En [27] se revisaron las aplicaciones de sistemas multiagente en microrred, desde las operaciones del mercado hasta la protección de microrred y la reposición de servicios. En [28] se presentó una prueba de concepto para suavizar la curva de carga utilizando un sistema de almacenamiento de energía con baterías (BESS por su sigla en inglés). En [29] se propuso un enfoque basado en la lógica difusa para un adecuado uso de los sistemas de almacenamiento de microrredes, y en [30] se estudió el impacto del sistema de almacenamiento en el costo operacional de una microrred típica. La utilización de los beneficios de los sistemas multiagente fue de un interés primordial en la gestión del almacenamiento de energía de las microrredes. En [31] también se centran en un agente de batería (y sus estrategias de administración de las mismas) que opera dentro de un marco de administración de microrred 
basado en sistemas multiagente, utilizando datos mínimos comunicados entre los componentes (agentes) de la red y sin usar predicción a largo plazo.

Todos los trabajos mencionados no contemplan la coordinación de baterías para múltiples microrredes interconectadas. En años recientes se vienen realizando investigaciones sobre la gestión de energía en escenarios donde las microrredes no están conectadas a la red principal.

En [32]-[34] se propone este enfoque basado en sistemas multiagente para una mejor gestión de la energía. Este asunto se encuentra en inicio de investigación y se pueden generar nuevos conocimientos relacionados con la interconexión y la gestión de energía en microrredes aisladas. En este artículo se estudia un sistema de gestión de energía basado en multiagentes para múltiples microrredes aisladas y aporta un modelo de estudio donde no son utilizados generadores diésel ni conexión a la red eléctrica principal. En la sección 2 se describe la metodología utilizada en el presente trabajo de investigación; y en la sección 3 se exponen los resultados obtenidos. Finalmente, en la sección 4 se presentan las conclusiones obtenidas, así como los posibles trabajos futuros.

\section{METODOLOGíA}

Para la simulación se han utilizado los datos de trabajos anteriores, en búsqueda de una estrategia que permita coordinar un sistema de microrredes compuesto. La metodología desarrollada se presenta por medio de las siguientes etapas (ver Figura1).

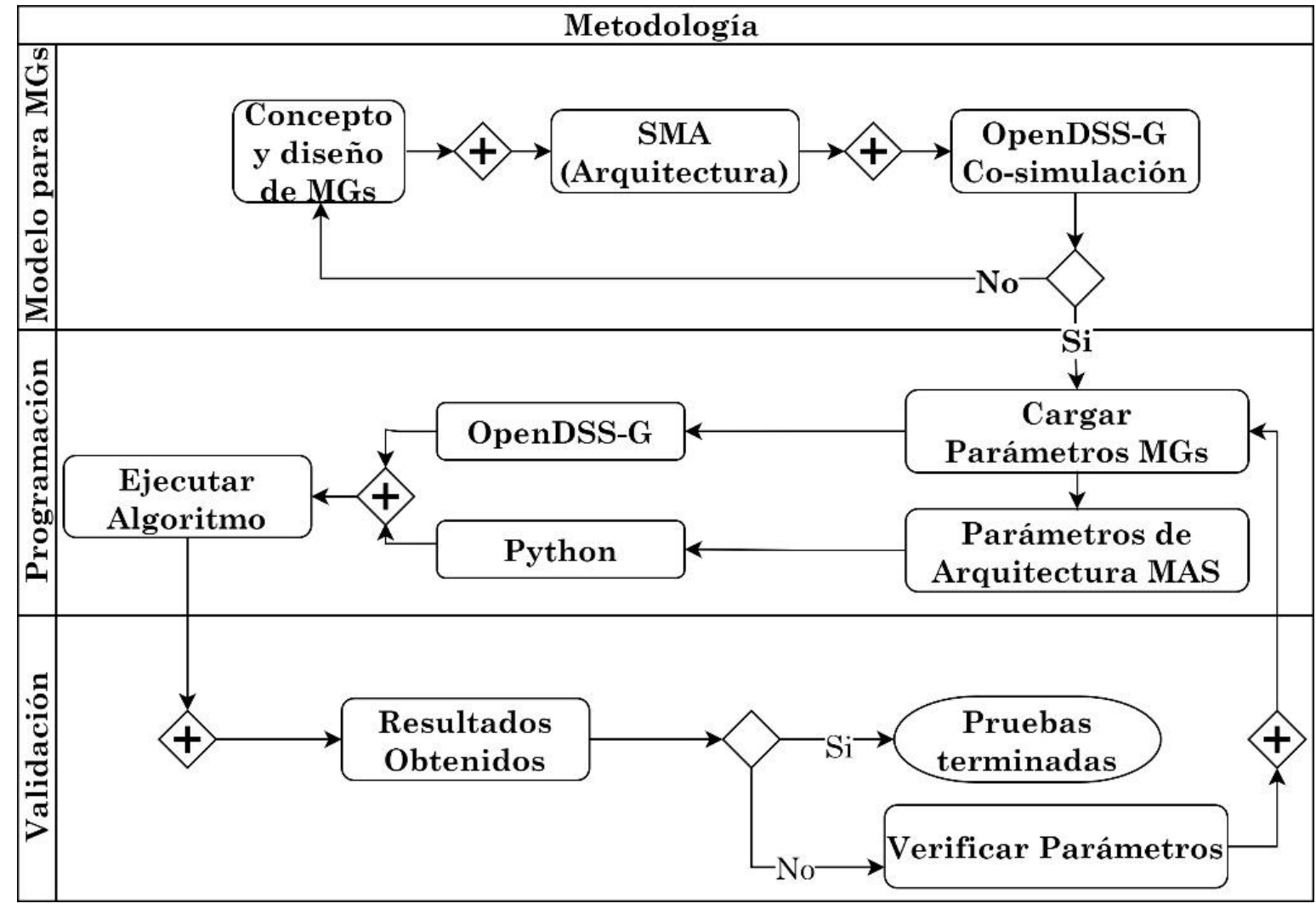

Figura 1. Diagrama de flujo etapas de la metodología. Fuente: elaboración propia. 


\subsection{Concepto y diseño}

En este artículo se observa la simulación de cuatro microrredes. Utilizando un enfoque de agente inteligente, se espera que una microrred suministre su demanda local de forma independiente; sin embargo, la carga y la intermitencia de la generación pueden provocar un exceso o falta de energía temporal de una microrred. Se sugiere en la literatura que este problema se puede mitigar almacenando energía en bancos de baterías y/o importando la energía requerida de una o más microrredes vecinas, después de su acoplamiento temporal [32], [33]. Esta gestión de la energía se puede realizar a través de la implementación de múltiples agentes. El desempeño de la estrategia desarrollada se evalúa mediante simulación en OpenDSS-G /Python.

Para propósitos de este estudio, el sistema multiagente es independiente de las arquitecturas de agrupamiento de las microrredes [32]-[34]. En la Figura 2 se observa el modelo de simulación a realizar.

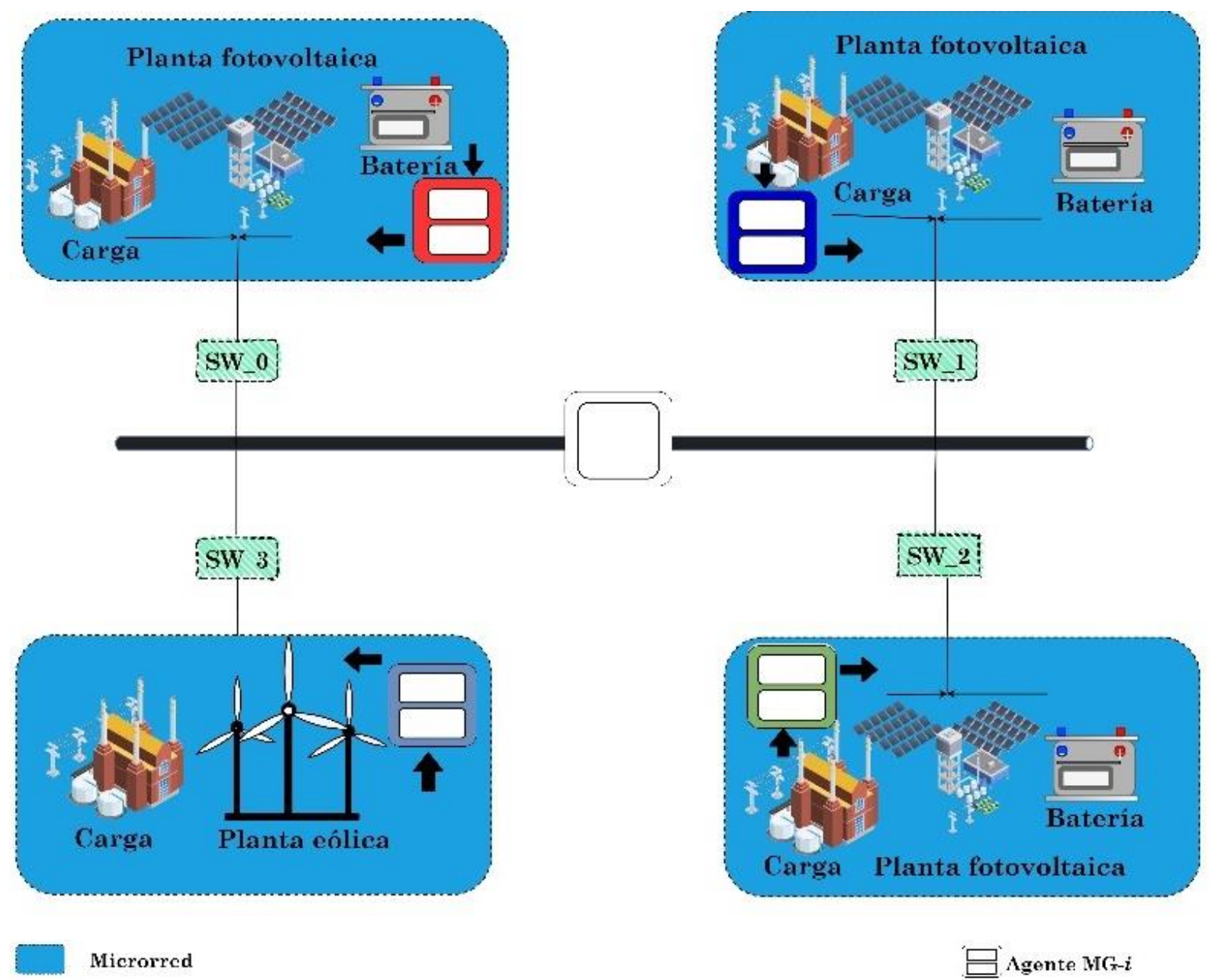

Figura 2. Esquema de microrredes para simulación. Fuente: elaboración propia.

\subsection{Sistemas multiagentes (MAS)}

Un agente inteligente es una entidad autónoma que monitorea el entorno a través de sensores y actúa sobre él mediante el uso de actuadores, dirigiendo su actividad al logro de los objetivos del agente [35]. El agente inteligente procesa la información recibida, hace 
comparaciones con reglas concretas, investiga posibles decisiones y finalmente selecciona la solución apropiada mientras se comunica con otros agentes en un entorno específico [36].

\subsection{OpenDSS-G}

OpenDSS-G es la interfaz gráfica del simulador de sistemas de distribución de código abierto del Instituto de Investigación de Energía Eléctrica (EPRI por su sigla en inglés), llamado OpenDSS, el cual ha evolucionado hacia un software de procesamiento en paralelo: OpenDSS V8. EPRI identificó la necesidad de desarrollar una interfaz para evaluar los futuros requisitos de redes inteligentes para estudios de planificación y operación.

OpenDSS-G es una interfaz integral para facilitar el uso de las funciones avanzadas de OpenDSS, proporcionando un entorno de desarrollo que imita una sala de control rodeada de herramientas gráficas que pueden ser interpretadas intuitivamente por los usuarios [37].

\subsection{Cosimulación de OpenDSS-G y Python}

Cada proceso de OpenDSS es operado y centralizado por los componentes que integra OpenDSS-G. Este proceso mantiene la conexión de simulación con todos los componentes de OpenDSS a través de un servidor TCP, incluidos los que están fuera de OpenDSS-G, como las plataformas de cosimulación y sus códigos externos (LabVIEW, Matlab, Python). El protocolo por el cual se realiza la comunicación se llama DSTCP. Los comandos requeridos para la conexión con aplicaciones externas están documentados en la interfaz TCP/IP contenida en el manual de OpenDSS-G.

\subsection{Parámetros para las microrredes}

Para la implementación del modelo mostrado en la Figura 2, se toma como microrred (MG por su sigla en inglés) un sistema conformado por paneles solares, baterías y un tipo de carga (hogar). Este es el caso de las microrredes 1, 2 y 3. Para la microrred 4, el sistema está conformado por un generador eólico y una carga que simula el consumo energético de un hogar, en este escenario no se tienen baterías para almacenamiento de energía (ver Figura 2 y Figura 4). Las microrredes se encuentran interconectadas por medio de un interruptor a un bus principal, con un voltaje generado por las microrredes de $120 \mathrm{~V}$ RMS y frecuencia constante de $60 \mathrm{~Hz}$. El control de los interruptores se realiza mediante comandos desde Python. Estas estructuras para la interconexión de microrredes es tema de estudio [33], [38].

En la Tabla 1 se describen los parámetros de potencia para las microrredes. Los datos de irradiancia solar, velocidad del viento y demanda de carga fueron obtenidos y ajustados de [39]-[41]. Los perfiles solares hacen referencias a las ciudades de Valledupar, para la MG-1; Sincelejo, para MG-2; Barranquilla, para la MG-3 y perfil eólico; y para la Guajira, MG-4, con un tiempo de toma de datos durante 24 horas. En la Figura 3 se observan los perfiles obtenidos. 
Tabla 1. Parámetros de operación para las microrredes. Fuente: elaboración propia.

\begin{tabular}{ccc}
\hline Variable & Significado de la variable & Valores límites \\
\hline Pv1 & Panel Solar MG-1 & $0 \leq \mathrm{Pv} 1 \leq 5 \mathrm{~kW}$ \\
Pv2 & Panel Solar MG-2 & $0 \leq \mathrm{Pv} 2 \leq 6 \mathrm{~kW}$ \\
Pv3 & Panel Solar MG-3 & $0 \leq \mathrm{Pv} 3 \leq 6.3 \mathrm{~kW}$ \\
Pe & Sistema de generación eólica & $0 \leq \mathrm{Pe} \leq 3.3 \mathrm{~kW}$ \\
Bt1 & Batería 1 & $0 \leq \mathrm{Bt} 1 \leq 5 \mathrm{~kW}$ \\
Bt2 & Batería 2 & $0 \leq \mathrm{Bt} 2 \leq 7.6 \mathrm{~kW}$ \\
Bt3 & Batería 3 & $0 \leq \mathrm{Bt} 3 \leq 7.7 \mathrm{~kW}$ \\
SOC1 & Estado de carga Batería 1 & $0.3 \leq \mathrm{SOC} 1 \leq 0.8$ \\
SOC2 & Estado de carga Batería 2 & $0.3 \leq \mathrm{SOC} 2 \leq 0.8$ \\
SOC3 & Estado de carga Batería 3 & $0.3 \leq \mathrm{SOC} 3 \leq 0.8$ \\
C1 & Carga 1 & $0 \leq \mathrm{C} 1 \leq 2.5 \mathrm{~kW}$ \\
C2 & Carga 2 & $0 \leq \mathrm{C} 2 \leq 2.4 \mathrm{~kW}$ \\
C3 & Carga 3 & $0 \leq \mathrm{C} 3 \leq 2.7 \mathrm{~kW}$ \\
C4 & Carga 4 & $0 \leq \mathrm{C} 4 \leq 2.5 \mathrm{~kW}$ \\
\hline
\end{tabular}


Perfil Solar Barranquilla - - Carga No.3 Perfil Eólico Guajira - - Carga No.4

Figura 3. Potencia generada y consumida para las microrredes (MG-i). Fuente: elaboración propia. 


\subsection{Arquitectura y diseño del sistema multiagente (MAS)}

La arquitectura diseñada está formada por cinco agentes inteligentes, uno por cada microrred y uno ubicado en el bus principal, como se presenta en la Figura 4. Para describir el MAS es necesario precisar las percepciones, acciones, el objetivo y el entorno. En la Tabla 2 se describen estos aspectos.



Figura 4. Topología de las microrredes (MG-i) y arquitectura de los agentes. Fuente: elaboración propia.

Tabla 2. Comportamiento de los agentes. Fuente: elaboración propia.

\begin{tabular}{cl}
\hline \multirow{2}{*}{ Percepciones } & $\begin{array}{l}\text { Potencia producida y consumida por las unidades de la microrred (Pv, Pw y SOC), } \\
\text { estado de los interruptores, conexión con las microrredes, rango de operación de los } \\
\text { bancos de baterías. }\end{array}$ \\
\hline Comportamiento & $\begin{array}{l}\text { Determinar los puntos de operación de los componentes del sistema. } \\
\text { Objetivos }\end{array}$ \\
$\begin{array}{l}\text { Satisfacer plenamente las demandas de energía y el estado de carga (SOC) de los } \\
\text { bancos de baterías con el objetivo de garantizar una mayor vida útil de las baterías. }\end{array}$ \\
Entorno & Todos los componentes de la microrred y microrredes vecinas. \\
Acción & Apertura y cierre de los interruptores. \\
\hline
\end{tabular}

En la Figura 4 y Figura 5 se puede observar que el modelo cuenta con cinco agentes (rojo, azul, verde, azul claro y blanco) que controlan y monitorean la potencia generada y el estado de las baterías (SOC) en cada microrred. Estos agentes se identifican como: agente MG-1 
(agente fotovoltaico No. 1), el agente MG-2 (agente fotovoltaico No. 2), el agente MG-3 (agente fotovoltaico No. 3), el agente MG-4 (agente eólico No. 4) y el agente GE (agente gestión de energía No. 5).

Los agentes inteligentes del sistema fueron informados sobre el estado de la microrred a través de monitores instalados, los cuales pueden asumir el rol de sensores. La topología del sistema de múltiples agentes se presenta en la Figura 4. La forma de comunicación entre todos los agentes es por TCP/IP. Toda la información es compartida entre todos los agentes.

Sin embargo, cada agente utilizó solo una parte de esa información, pues no todas las microrredes requerían o exportan la misma cantidad de potencia.

En la Figura 5 se puede revisar con mayor detalle la arquitectura del MAS. Los agentes fotovoltaicos (MG-i) tienen el mismo modo de operación. Estos agentes se conectan con los monitores de información para obtener datos de las potencias de las fuentes de energía renovables, el SOC y los consumos de energía en la carga (Ci). Esta información fue utilizada por los agentes No. 1, No. 2, No. 3 y No. 5. El agente eólico (MG-4) es autónomo, se conecta al bus principal la mayor parte del tiempo para entregar energía excedente o para conseguir energía para su carga local. Esta información fue utilizada por los agentes No. 1, No. 2, No. 3 y No. 5.

El agente (GE) realiza la gestión de energía tomando información de las microrredes vecinas para conocer la potencia excedente y la potencia actual de las baterías. Este agente envía información para que las microrredes realicen apertura o cierre de sus interruptores para cargar baterías. Esta información fue utilizada por los agentes No. 1, No. 2 y No. 3.
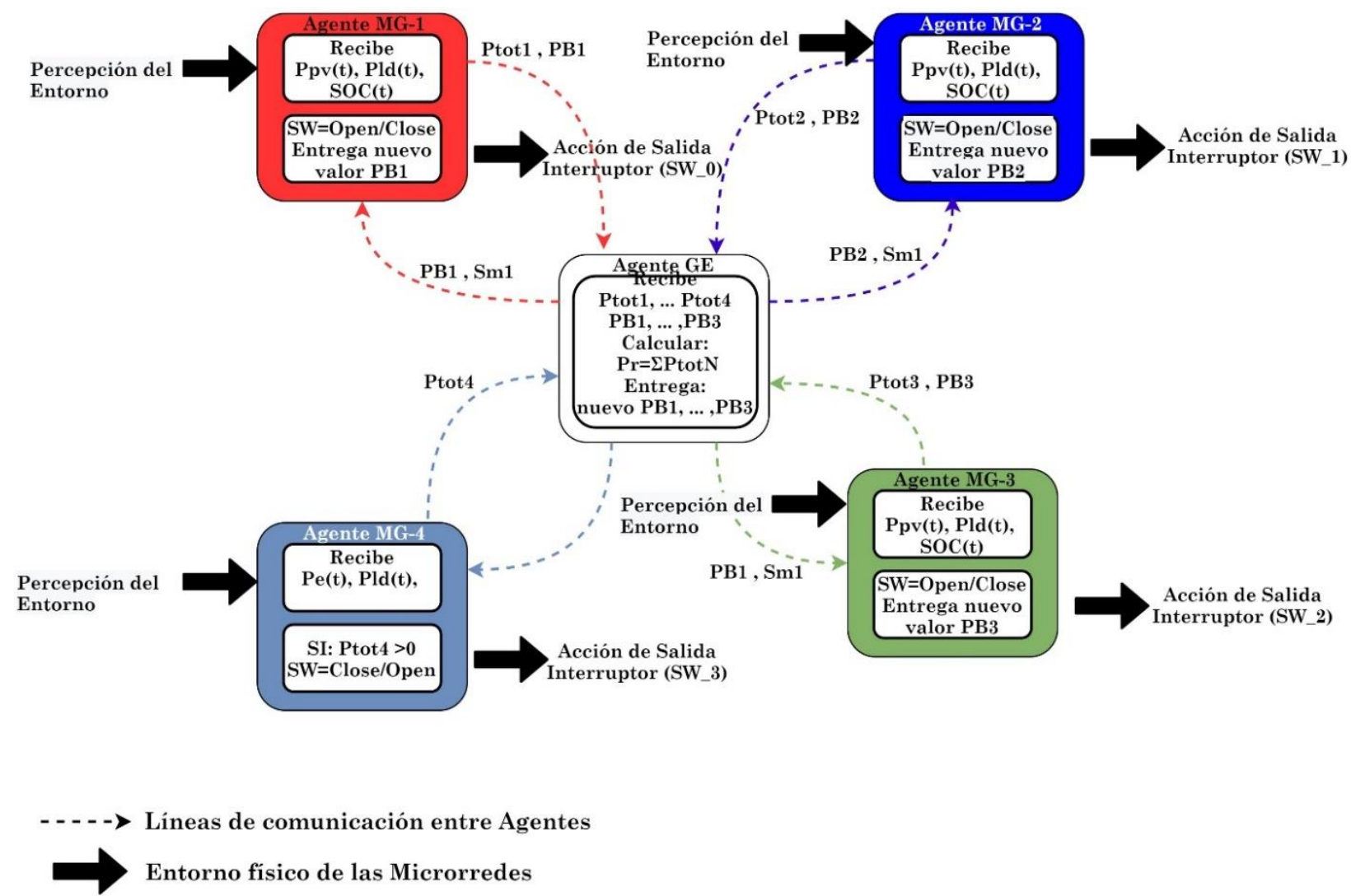

Figura 5. Arquitectura del Sistema multiagentes (MAS). Fuente: elaboración propia. 


\subsection{Algoritmo del sistema multiagente (MAS)}

Para el diagrama del agente fotovoltaico mostrado en la Figura 6, el algoritmo recibe información de la potencia generada en la DER, la potencia de consumo de la carga y el SOC de la batería. El primer paso realizado por el algoritmo es adquirir la diferencia de potencia que hay en el panel solar y la potencia consumida por la carga. Una vez obtenida la diferencia de potencia, verifica el SOC de la batería, el cual debe estar entre los valores de estado de carga (SOC_MIN) y (SOC_MAX), para los valores mínimo y máximo, teniendo en cuenta los trabajados en [32]. Dependiendo del estado de carga (SOC), el algoritmo calcula la potencia total que se está generando en ese momento en la MG-i. Este valor calculado es entregado al agente de gestión de energía (GE), y dependiendo del estado de la batería se le puede indicar al agente (GE) que se necesita cargar o descargar, o mantenerse con la reserva. Si el SOC de la batería es mayor al valor SOC_MAX, el agente fotovoltaico no carga la batería con el fin de evitar un exceso de carga, caso contrario si es menor indica que requiere energía. Si el SOC es mayor al valor SOC_MIN, el algoritmo permite la descarga de la batería hasta el valor mínimo. Una vez alcanzado este valor no se permite continuar la descarga, esto con el fin de evitar descargas profundas en la batería. Después de enviar información al agente de gestión de energía, la información de regreso al agente fotovoltaico es abrir o cerrar su interruptor principal para entregar potencia a la microrred y permitir la carga o descarga de la batería.

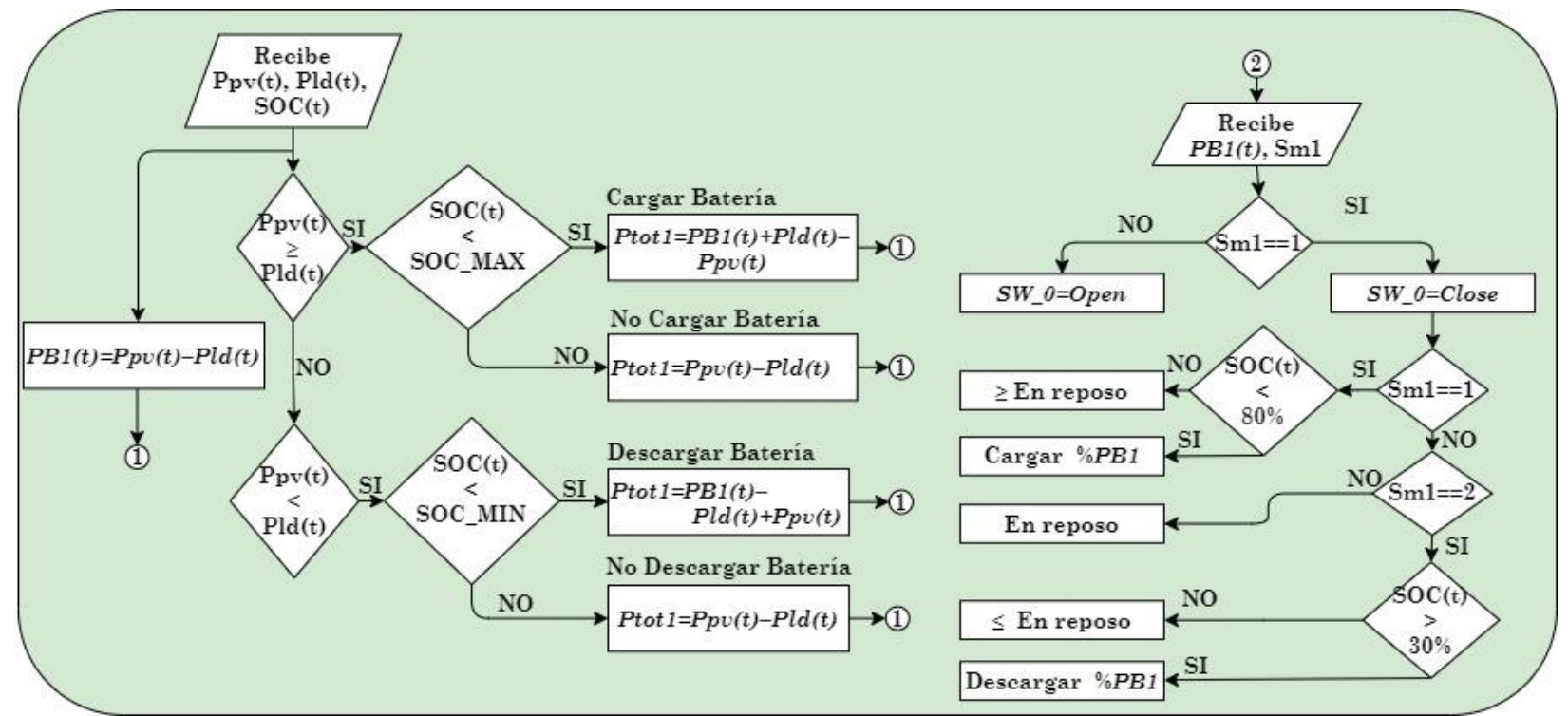

Figura 6. Algoritmo agente fotovoltaico (MG-1,2,3). Fuente: elaboración propia.

El algoritmo del agente de gestión de energía (GE), presentado en la Figura 7, es el encargado de realizar el cálculo de potencia entre las cuatro microrredes. Este algoritmo obtiene información sobre la cantidad de potencia que generan las MG. El algoritmo recibe información de la potencia total de cada MG-i y la potencia actual de las baterías. Una vez recibida esta información ejecuta el cálculo de la potencia total; de esta potencia se toma un nuevo valor de potencia requerido por las baterías. Luego de que se tiene el resultado del cálculo, el algoritmo informa al agente de la microrred correspondiente que puede abrir o cerrar interruptores para el paso de potencia requerida. En la Tabla 3 se pueden apreciar todas las variables asociadas en el algoritmo. 


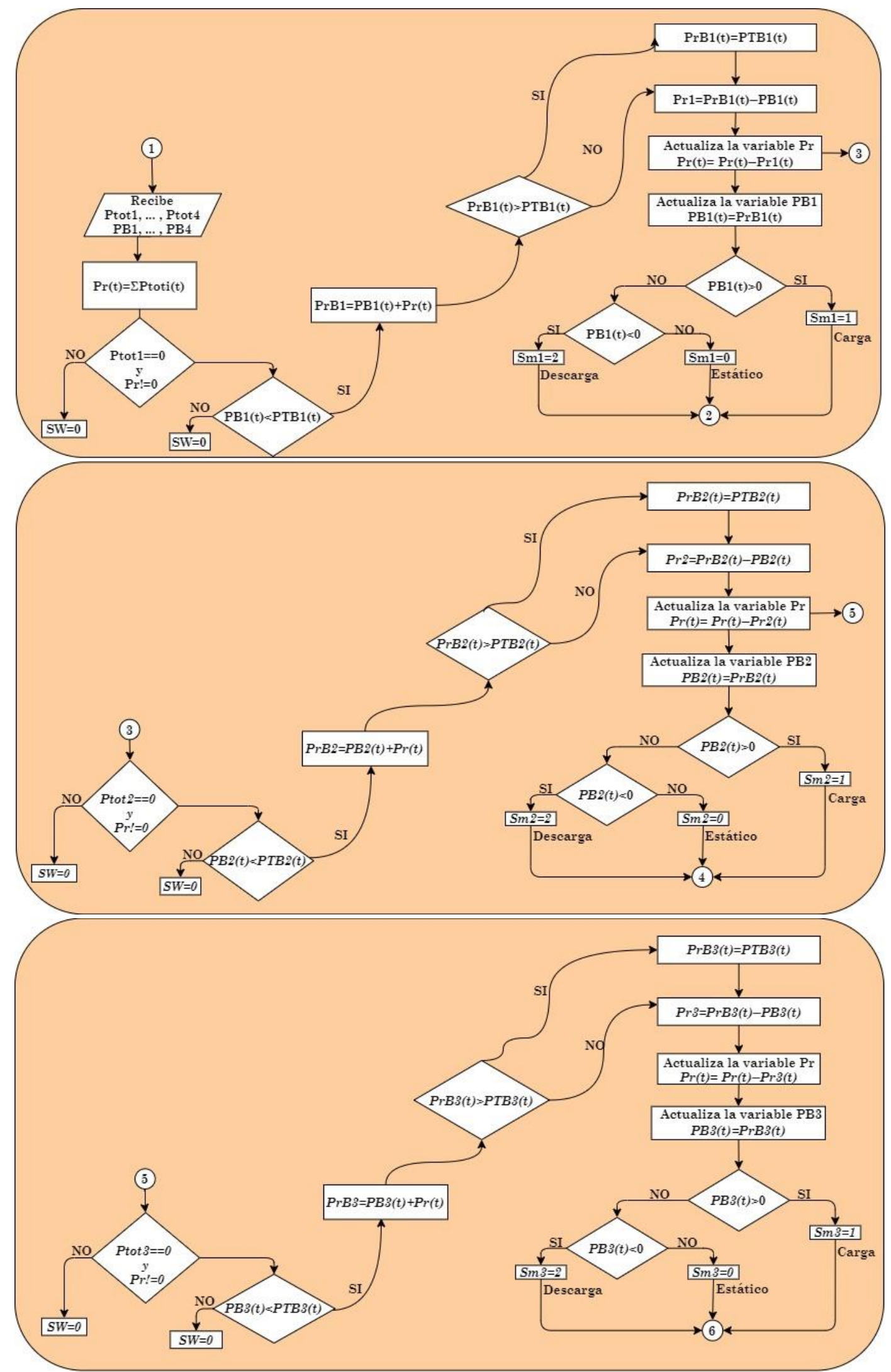

Figura 7. Algoritmo para agente de gestión de energía GE. Fuente: elaboración propia. 
Tabla 3. Variables utilizadas en el algoritmo. Fuente: elaboración propia.

\begin{tabular}{llll}
\hline Variable & Significado de la variable & Variable & Significado de la variable \\
\hline Ppv & Potencia generada por los paneles & $\mathrm{Sm} 0$ & No cargar baterías \\
Pld & Potencia generada por la carga & $\mathrm{Sm} 1$ & Cargar baterías \\
Ptoti & Potencia residual en una MG & $\mathrm{Sm} 2$ & Descargar baterías \\
SOC_MIN & Estado de carga mínimo batería & $\mathrm{PTB} i$ & Potencia máx. de la batería \\
SOC_MAX & Estado de carga máximo batería & $\mathrm{Pr}$ & Potencia residual de todas las MG \\
PBi & Potencia batería & $\mathrm{SW} i$ & Interruptor de conexión al bus \\
\hline
\end{tabular}

En la Figura 8 se describe el algoritmo del agente eólico. Su objetivo principal es mantener la potencia requerida para la carga No. 4, contrario a los agentes fotovoltaico, este no tiene baterías. El algoritmo del agente eólico tiene la libertad de conectarse al bus principal para entregar energía o para tomar energía requerida. Por el escenario del perfil la mayor parte del tiempo entrega energía.

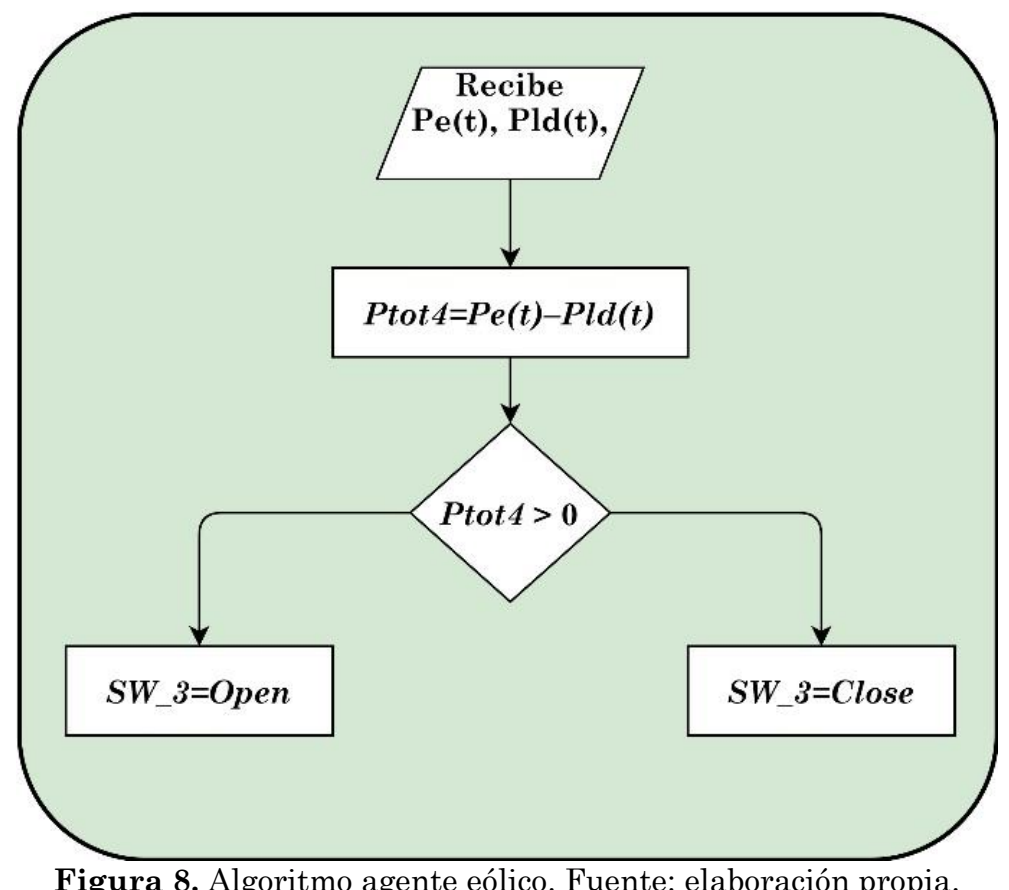

Figura 8. Algoritmo agente eólico. Fuente: elaboración propia.

\section{RESULTADOS}

Se probaron diferentes combinaciones de DER, baterías y cargas durante el proceso de gestión de energía. Se realizó la simulación de dos casos para ilustrar en detalle el perfil de carga, el estado de carga de las baterías y el proceso de despacho de las DER. Estos eventos importantes están acompañados de interruptores.

\subsection{Caso de prueba 1: operación en modo isla}

Este primer caso de prueba planteó un escenario en donde la energía producida es mayor a la cantidad de energía requerida. Este es el escenario ideal para la operación en modo isla, 
es decir que no hay interconexión entre las microrredes. En la Tabla 1 se indican las DER, las baterías y las cargas utilizadas para este caso. Los perfiles de potencia de las cargas y las potencias generada por las DER se pueden apreciar en la Figura 3. Los datos fueron obtenidos y organizados de [39]-[41]. El estudio del caso de prueba 1 fue simulado durante 24 horas usando el modelo planteado en la Figura 1.

Para el estudio del caso se toman tres resultados por cada microrred (MG-1, MG-2, MG 3). En las Figuras 9, 10 y 11 se observa el comportamiento de la potencia generada en los paneles solares, las baterías y el consumo de una carga doméstica. En cada una de estas gráficas se aprecia la respuesta de seguimiento que realizan las baterías para cumplir con el consumo eléctrico de la carga en las horas donde los paneles no generan potencia. El comportamiento que tienen las baterías, según la gráfica, es: para valores negativos está cargado y para valores positivos se descarga. El agente programado para cada microrred tiene dentro de sus objetivos que las baterías proporcionen potencia de acuerdo con el comportamiento de consumo de la carga.

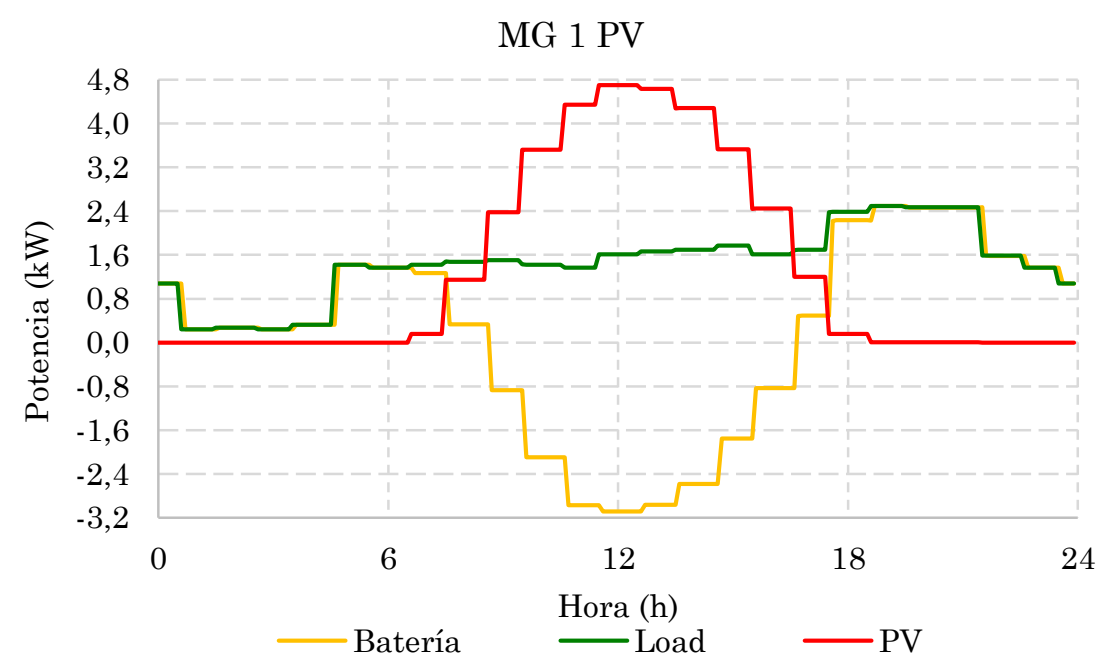

Figura 9. Potencia MG-1 para caso de prueba en isla. Fuente: elaboración propia.

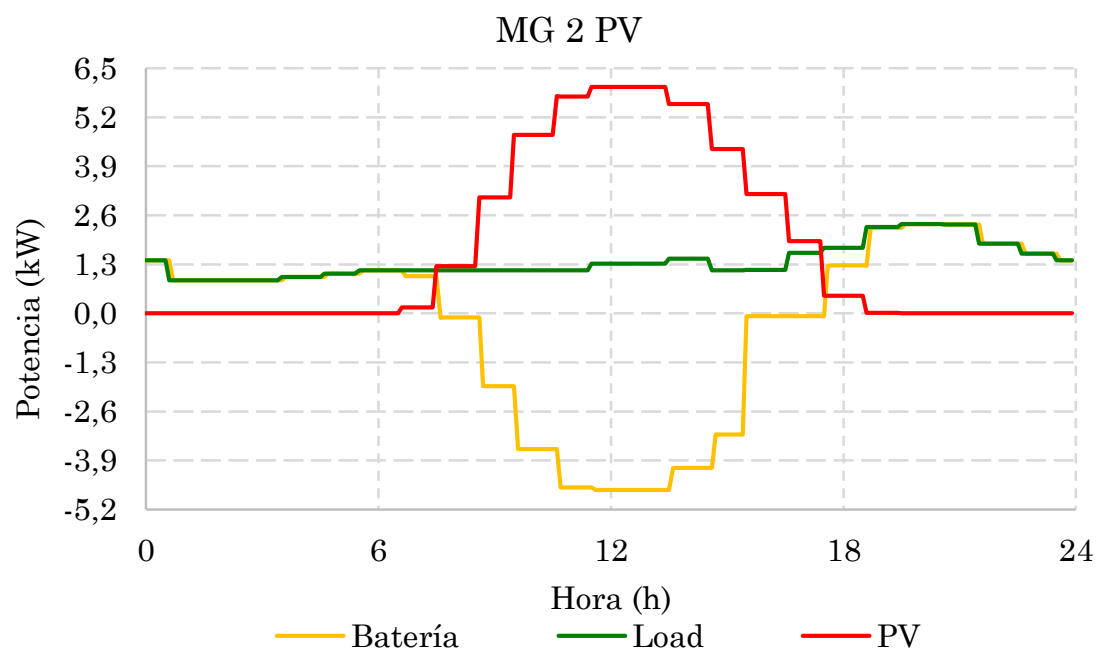

Figura 10. Potencia MG-2 para caso de prueba en isla. Fuente: elaboración propia. 


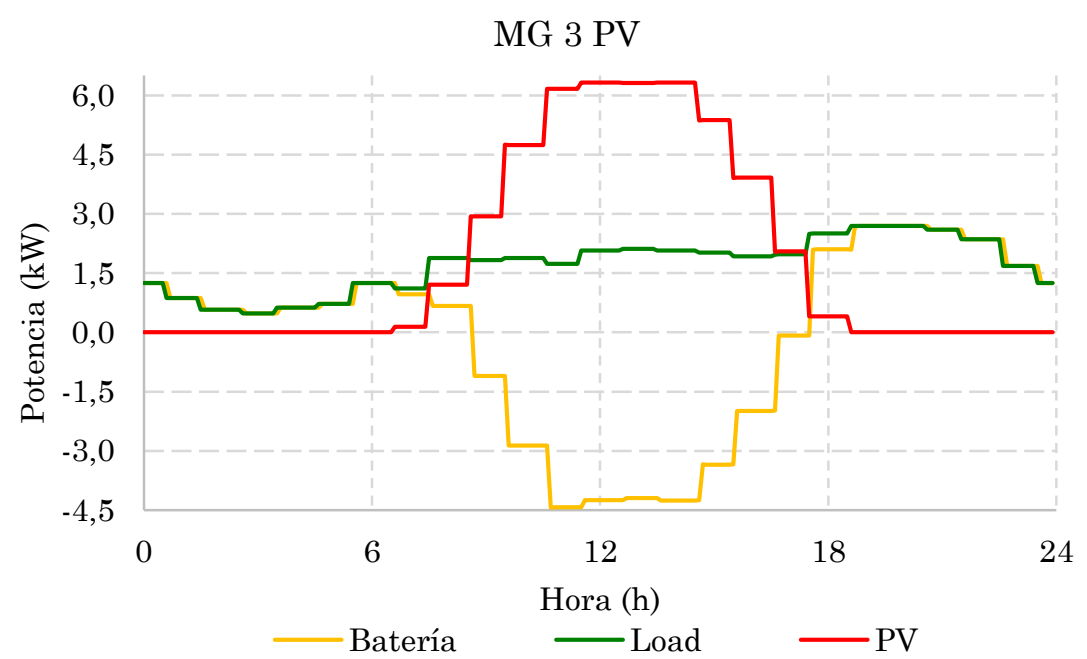

Figura 11. Potencia MG-3 para caso de prueba en isla. Fuente: elaboración propia.

En los resultados de los perfiles de voltaje, como se observa en las Figuras 12, 13 y 14, este presenta variaciones, pero se mantiene dentro del rango de los $108 \mathrm{~V}$ RMS. En las gráficas del estado de carga de las baterías (SOC), mostrado en las Figuras 15, 16 y 17, se observa que estas se mantienen en un rango entre el $30 \%$ y el $80 \%$ de su capacidad. Se puede apreciar en estas gráficas que durante las horas 6 hasta las 16 ocurre un incremento en las curvas hasta lograr el máximo de carga permitido; esto ocurre en las horas en las que actúan los paneles solares.



Figura 12. Voltaje Carga Ld_1 en la MG-1 para caso de prueba en isla. Fuente: elaboración propia. 




Figura 13. Voltaje Carga Ld_2 en la MG-2 para caso de prueba en isla. Fuente: elaboración propia.

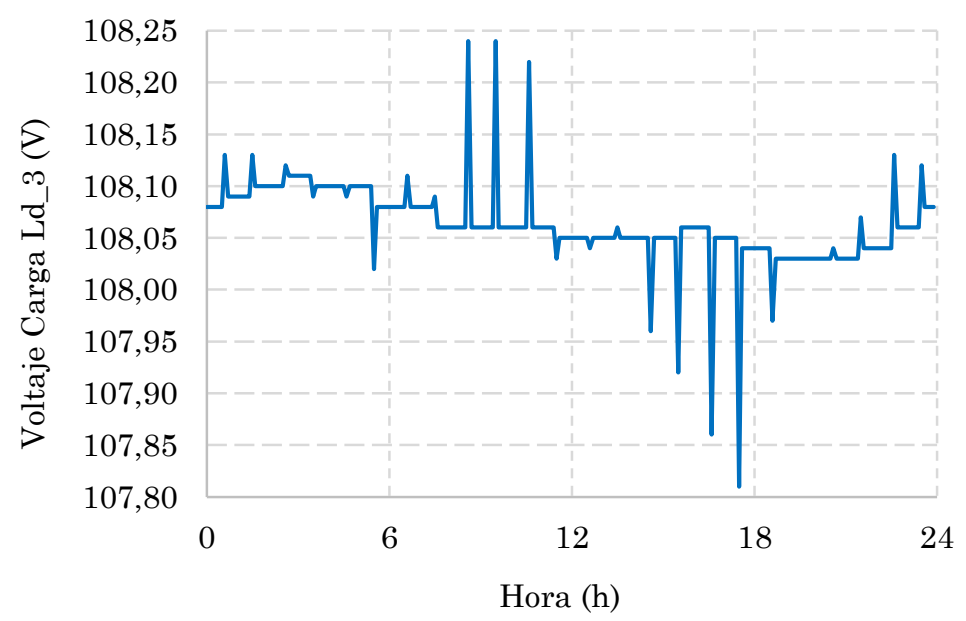

Figura 14. Voltaje Carga Ld_3 en la MG-3 para caso de prueba en isla. Fuente: elaboración propia.

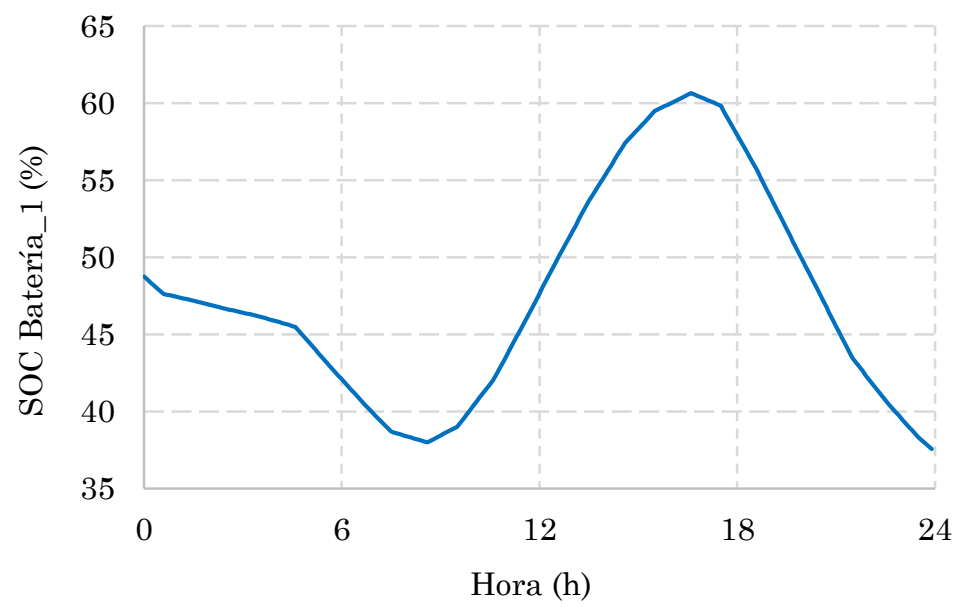

Figura 15. SOC Batería_1 en la MG-1 para caso de prueba en isla. Fuente: elaboración propia. 


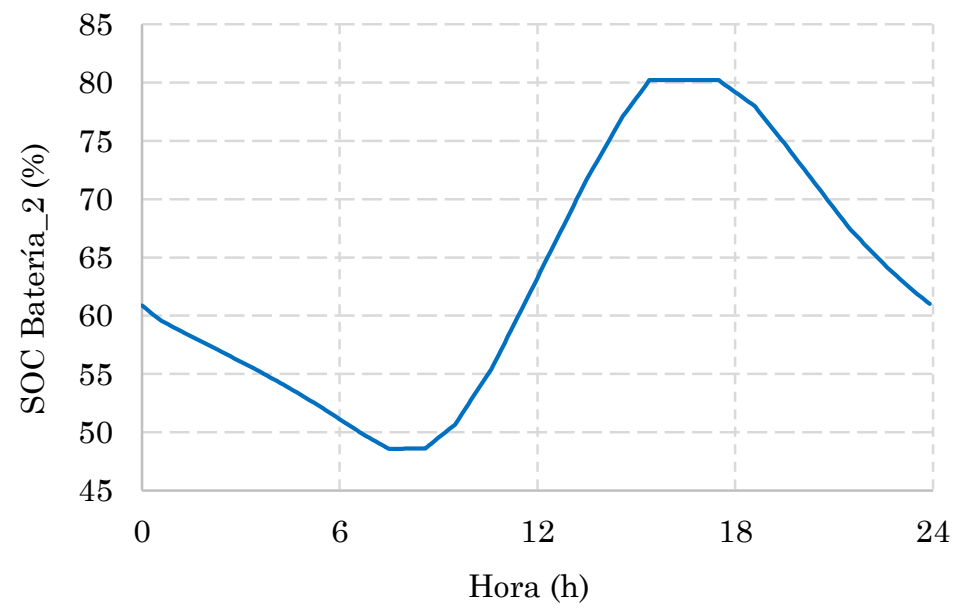

Figura 16. SOC Batería_2 en la MG-2 para caso de prueba en isla. Fuente: elaboración propia.

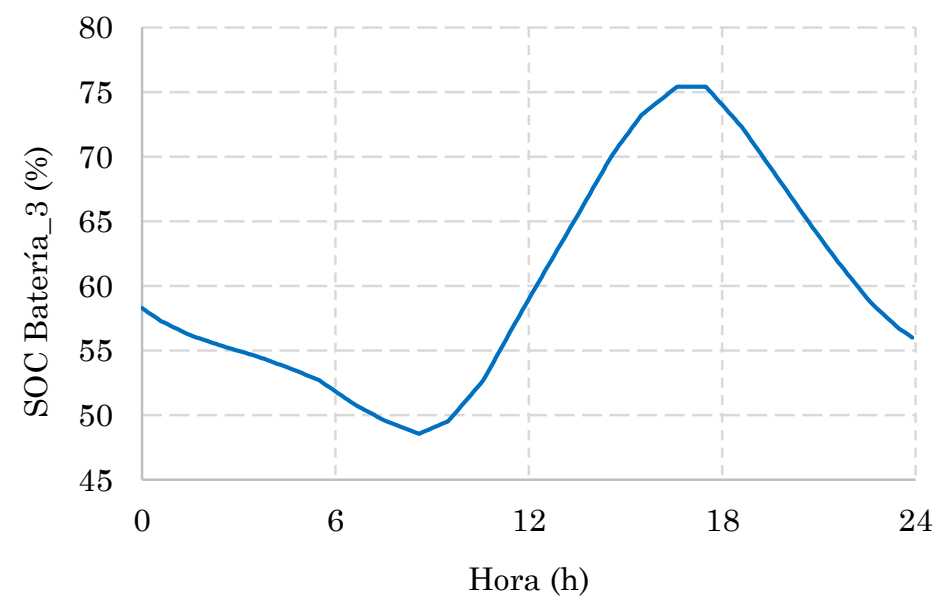

Figura 17. SOC Batería_3 en la MG-3 para caso de prueba en isla. Fuente: elaboración propia.

En la Figura 18, que corresponde a la MG-4, se muestran los resultados obtenidos de potencia generada por la fuente eólica y consumo eléctrico en la carga (C_4). Esta microrred (MG) no tiene banco de baterías, y abre y cierra el interruptor (SW_3) de manera autónoma. Por último, la Figura 19 muestra el voltaje RMS medido en el bus principal al estar las microrredes en modo isla, es decir, no interconectadas. Este valor es de $0 \mathrm{~V}$. 


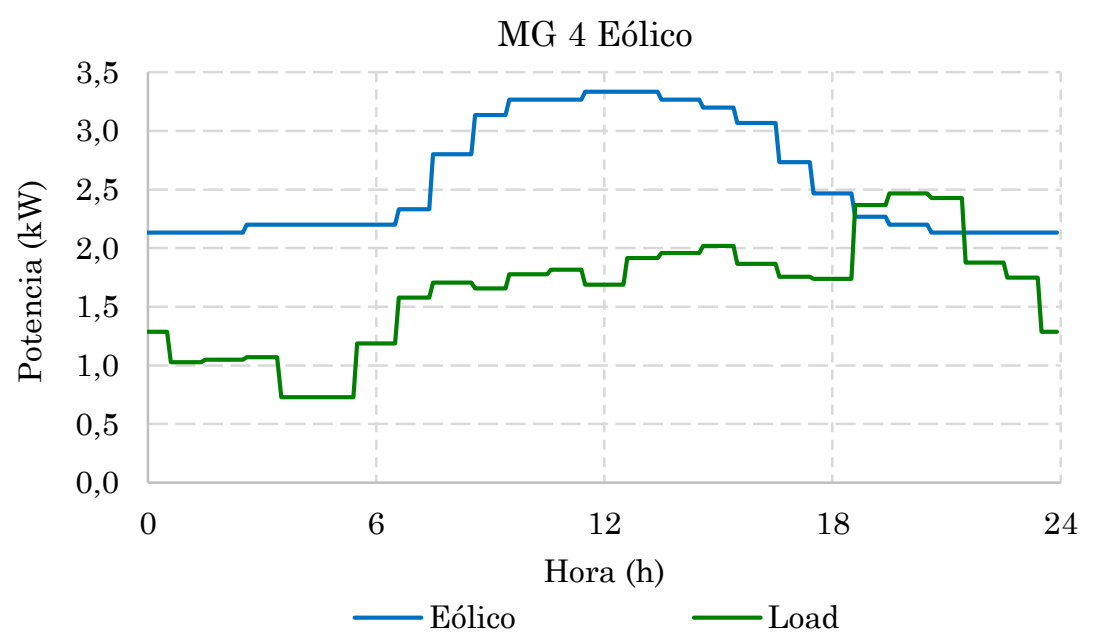

Figura 18. Potencia MG-4 para caso de prueba en isla. Fuente: elaboración propia.

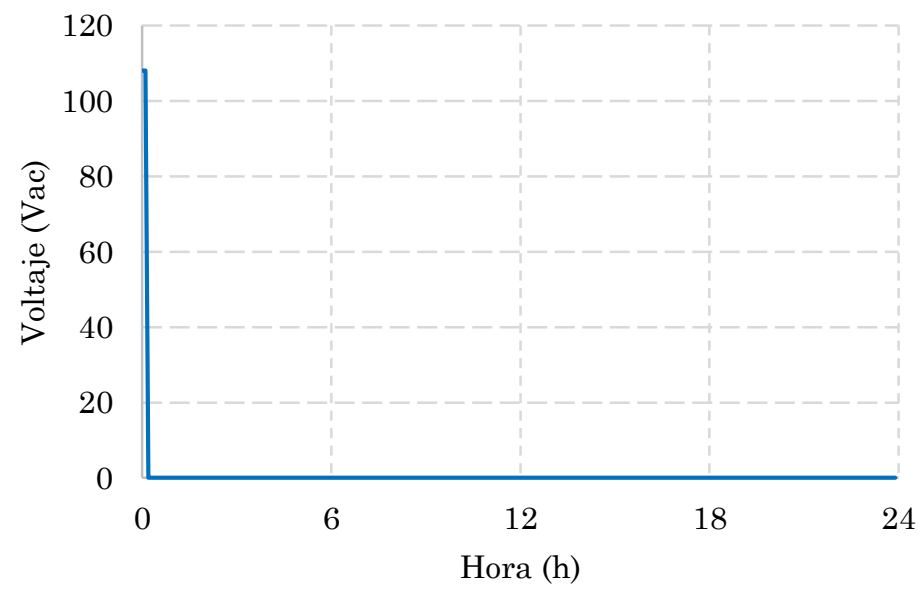

Figura 19. Voltaje en el bus principal del sistema para caso de prueba en isla. Fuente: elaboración propia. *La Figura 19 corresponde al voltaje A.C. medido en el bus de interconexión de todas las microrredes.

\subsection{Caso de prueba 2: interacción entre MG}

El segundo caso de prueba muestra un escenario en donde hay interconexión de todas las microrredes; los agentes programados presentan dentro de sus objetivos la decisión de apertura o cierre de sus interruptores (SW_i, por sus iniciales en inglés) para permitir el paso de potencia hacia el interior o exterior de la microrred. Como en el caso de prueba anterior, en la Tabla 1 se indicaron los DER, las baterías y las cargas utilizadas para este caso. En la Figura 3 se observaron los perfiles de potencia generados por las cargas eléctricas y las potencias generadas por los DER. Estos datos fueron tomados de [39]-[41]. Para el análisis del caso de prueba 2 , este fue simulado durante 24 horas usando el modelo mostrado en la Figura 1.

Para este caso de prueba 2 se tomaron tres resultados por cada microrred (MG-1, MG-2, MG-3). En las Figuras 20, 21 y 22 se puede analizar el comportamiento de la potencia. Por otro lado, en la Figura 20 se observa que en la MG-1 la batería no entrega energía de acuerdo con el consumo de potencia de la carga eléctrica; al estar en interacción con las demás 
microrredes aprovecha la energía del bus principal para suplir la demanda de carga; esto se observa en las horas 2 a 5, cuando la batería está cargando.

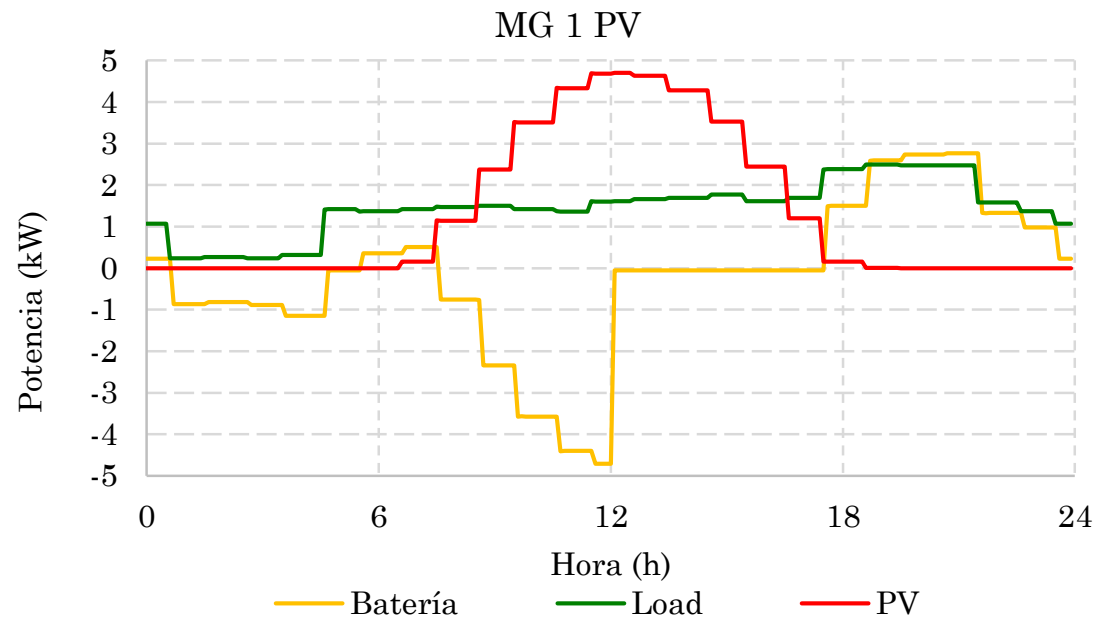

Figura 20. Potencia MG-1 para caso de prueba de interconexión. Fuente: elaboración propia.

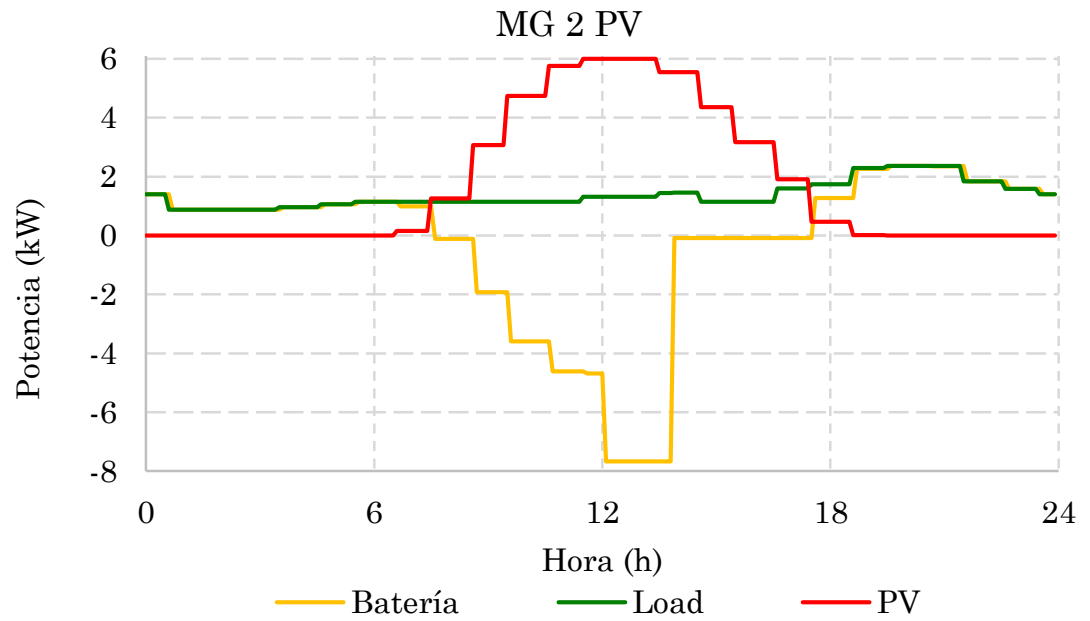

Figura 21. Potencia MG-2 para caso de prueba de interconexión. Fuente: elaboración propia.

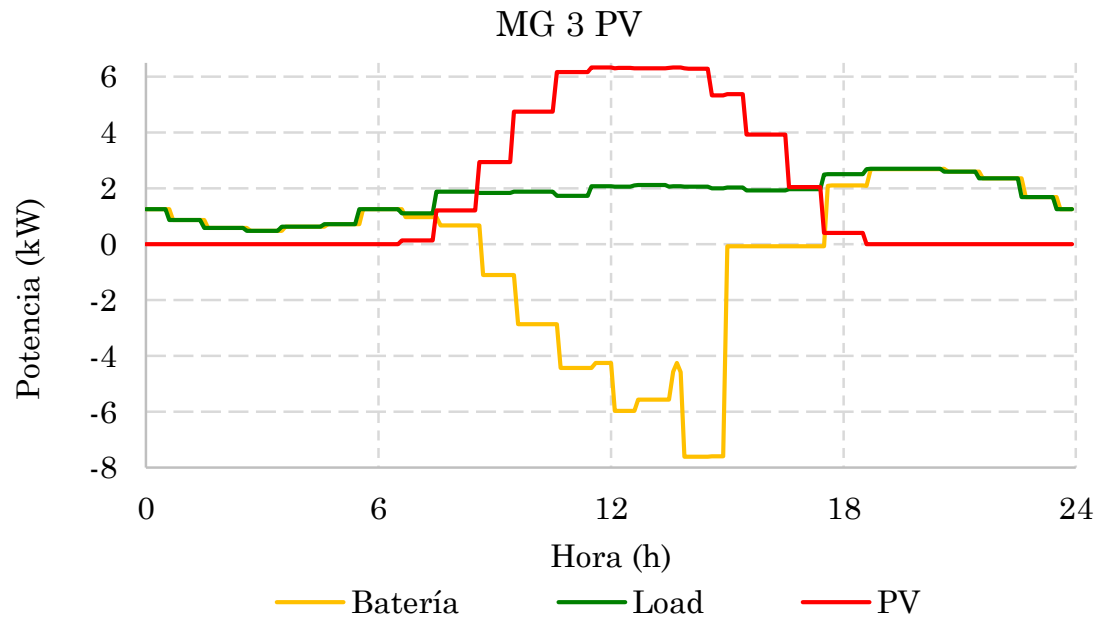

Figura 22. Potencia MG-3 para caso de prueba de interconexión. Fuente: elaboración propia. 
En la Figura 21 y 22 se muestra que para la MG-2 y MG-3, la batería entrega potencia de acuerdo con la necesidad de consumo de la carga eléctrica (C_i). Asimismo, en las Figuras 20, 21 y 22 se puede apreciar que durante las horas de trabajo los paneles solares y las baterías logran una estabilidad, luego de esto la gráfica se mantiene en cero, resultado que indica que la batería tiene su máxima capacidad de carga programada y debe mantenerse en estado de reposo hasta que se requiera la potencia almacenada.

Las Figuras 23, 24 y 25 se refieren a los perfiles de voltaje medidos, este se mantiene aproximadamente en 108 V RMS. De igual forma, en las Figuras 26, 27 y 28 se analizan los estados de carga de las baterías (SOC) y se observan cambios con respecto al caso de prueba en modo isla. En este sentido, el SOC de las baterías de prueba varía entre el 50 \% y el $80 \%$ sin pasar su máximo permitido o programado en estas circunstancias. Para este caso de prueba las baterías se cargan con mayor velocidad. La Figura 29 hace referencia a la MG-4, de generación eólica, la cual mantiene la respuesta igual que en el caso anterior. En las Figuras 30 y 31 se observa que el voltaje puede variar en el bus de 0 a $108 \mathrm{~V}$ RMS cuando es conectada una microrred o varias microrredes al mismo tiempo durante el periodo de interconexión.

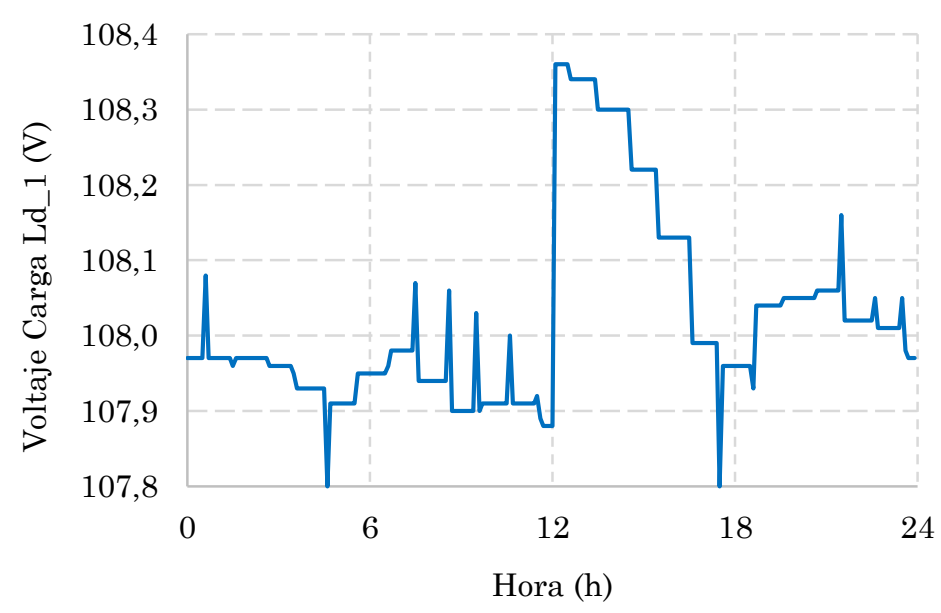

Figura 23. Voltaje Carga Ld_1 en la MG-1 para caso de interconexión. Fuente: elaboración propia.

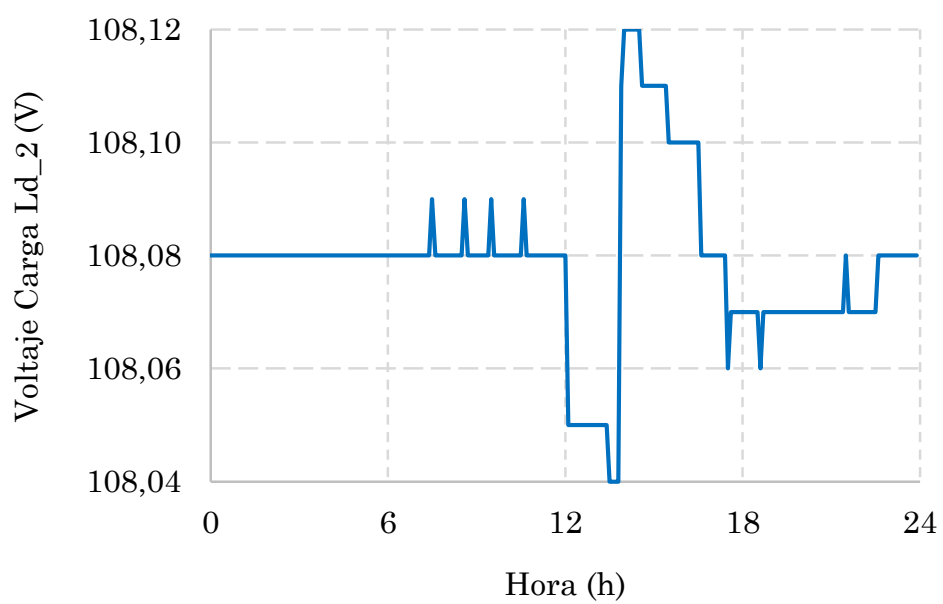

Figura 24. Voltaje Carga Ld_2 en la MG-2 para caso de interconexión. Fuente: elaboración propia. 


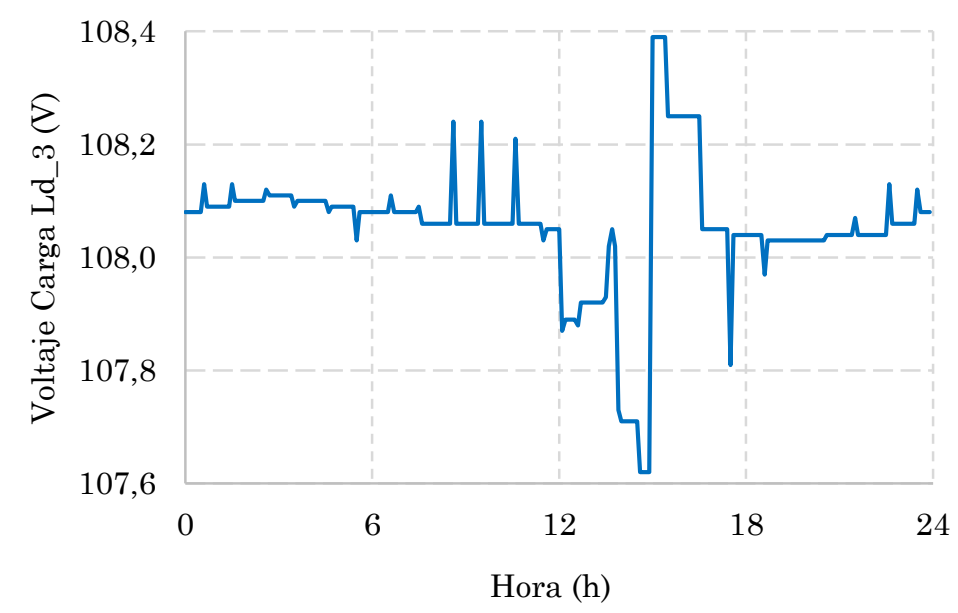

Figura 25. Voltaje Carga Ld_3 en la MG-3 para caso de interconexión. Fuente: elaboración propia.

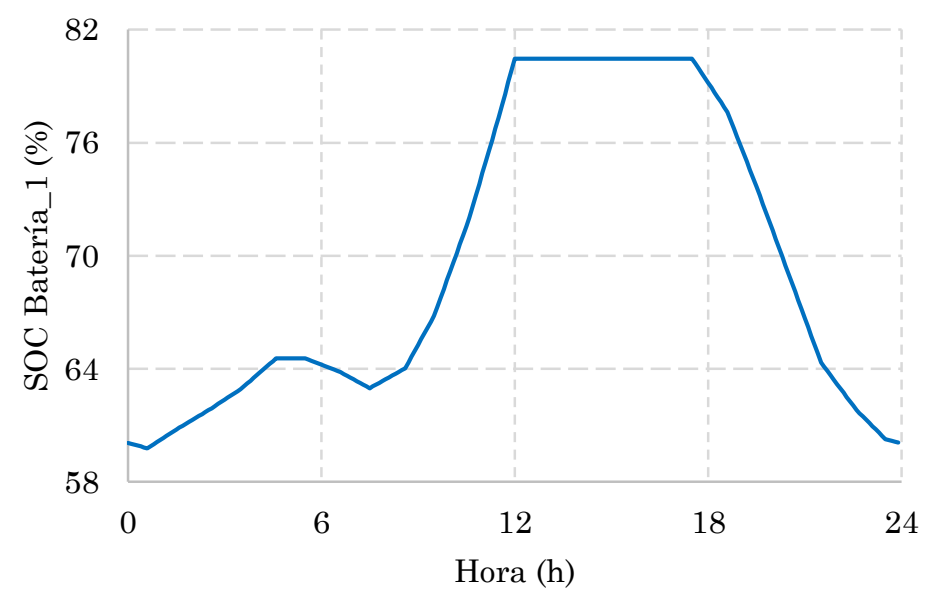

Figura 26. SOC Batería_1 en la MG-1 para caso de prueba de interconexión. Fuente: elaboración propia.

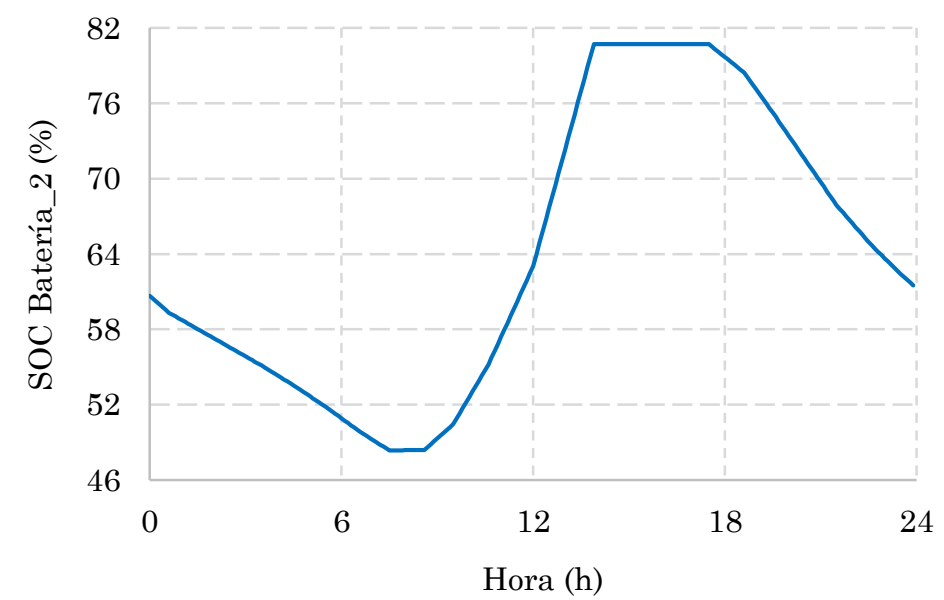

Figura 27. SOC Batería_2 en la MG-2 para caso de prueba de interconexión. Fuente: elaboración propia. 


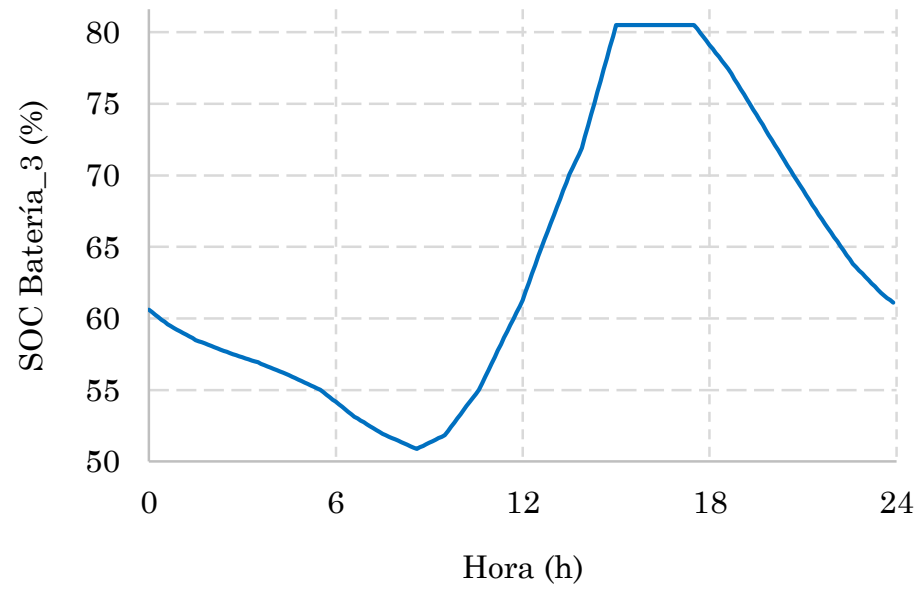

Figura 28. SOC Batería_3 en la MG-3 para caso de prueba de interconexión. Fuente: elaboración propia.

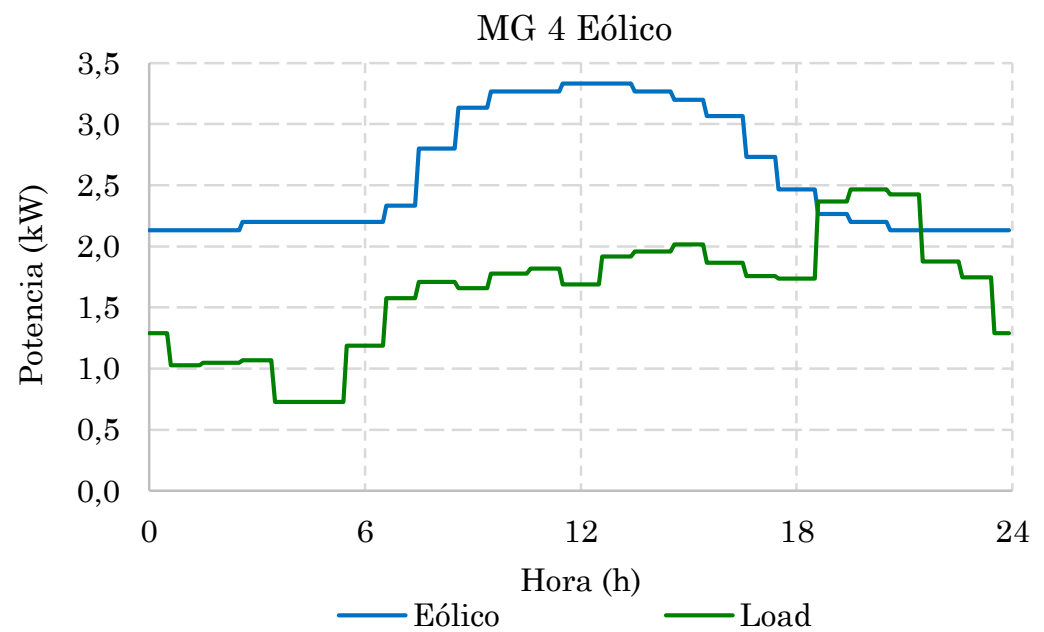

Figura 29. Resultados de potencia en la MG-4 para caso de prueba en interconexión. Fuente: elaboración propia.

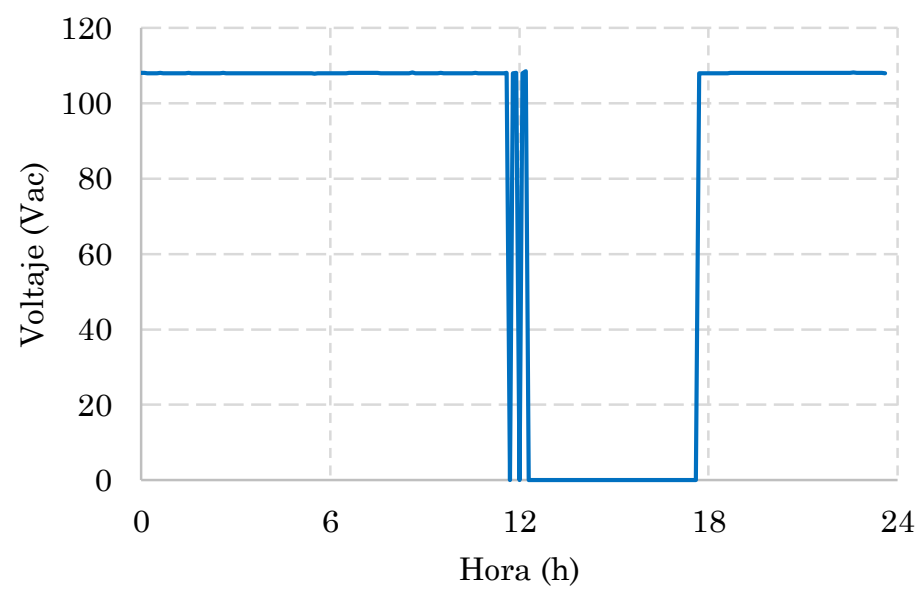

Figura 30. Conexión de una microrred (MG-3) al bus principal. Fuente: elaboración propia. 


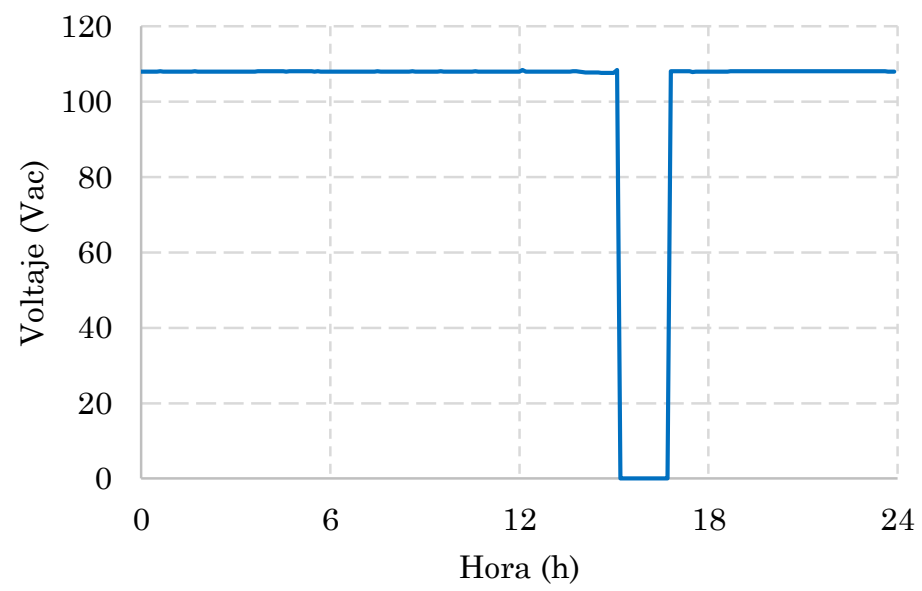

Figura 31. Interacción de todas las microrredes MG-i. Fuente: elaboración propia.

**Las Figuras 30 y 31 corresponde al voltaje A.C. medido en el bus de interconexión de todas las microrredes.

\section{CONCLUSIONES}

En este artículo se desarrolló una arquitectura aplicando sistemas multiagente que permiten gestionar el modo de operación de un sistema de microrredes distribuidas en un entorno aislado. Se describieron los detalles sobre el diseño y desarrollo del sistema de agentes múltiples con el objetivo de prevenir sobrecargas y profundas descargas en las baterías. Se han incorporado al sistema funcionalidades para aislar las microrredes. El sistema multiagente también implementa un algoritmo para soportar el intercambio de potencia entre las microrredes de forma independiente. La simulación del sistema se realizó mediante OpenDSS-G y Python.

De los resultados obtenidos se tiene que un sistema descentralizado de gestión de energía, basado en la teoría de sistemas de múltiples agentes, puede tener beneficios importantes, como, por ejemplo: el carácter autónomo de las microrredes, que un enfoque descentralizado utiliza mejor los dispositivos que uno centralizado $\mathrm{y}$, por último, que un sistema descentralizado de gestión energética es económicamente más competitivo. La ventaja más importante de usar una arquitectura descentralizada es que la microrred gestionada tiene muchas posibilidades de operación en caso de ausencia o excedente de potencia, además del control que se puede tener sobre la vida útil de las baterías. La aplicación de sistemas multiagente en las microrredes puede contribuir con un mayor nivel de generación eléctrica, en especial para las zonas no interconectadas, esto ayuda en la protección del medio ambiente al disminuir el uso de generadores diésel. Finalmente, con la teoría de multiagente se pueden crear sistemas de generación distribuida más confiables debido a su capacidad autónoma de tomas de decisiones, para cubrir demandas eléctricas desde microrredes vecinas. Otros de los aportes que brinda este artículo es la implementación de un modelo de estudio donde no son utilizados generadores diésel ni conexión a la red eléctrica principal, logrando concluir, desde los resultados, la importancia de las baterías para lograr la estabilidad eléctrica del sistema.

Con el propósito de seguir investigando sobre la gestión de energía y la interconexión de microrredes aisladas, se propone desarrollar un algoritmo más eficiente para la optimización y coordinación de los agentes múltiples que pueda seleccionar la opción más adecuada para la conexión entre las microrredes que efectúe el análisis del flujo de potencia (voltajes y 
frecuencia). De igual forma se puede implementar un sistema multiagente basado en sistemas embebidos que permita interactuar los agentes con OpenDSS-G.

\section{AGRADECIMIENTOS}

A los grupos de investigación y a la Facultad de Ingeniería Electrónica de la Universidad Santo Tomás. El proyecto no contó con financiamiento de institución pública o privada.

\section{CONFLICTOS DE INTERÉS DE LOS AUTORES}

Los autores declaran no tener ningún conflicto de interés.

\section{CONTRIBUCIÓN DE LOS AUTORES}

Alfredo Sánchez Silvera. Diseño, programación de los algoritmos, ejecución de los casos de pruebas, desarrollo de la investigación y redacción del documento.

José Guillermo Guarnizo Marín. Desarrollo de la investigación, diseño, redacción, revisión y aprobación final del artículo.

Edwin Francisco Forero García. Contribuye con los conceptos eléctricos de funcionamiento, y con la revisión y aprobación final del artículo.

Davis Montenegro Martínez. Contribuye con información del funcionamiento del software OpenDSS-G, interfaz gráfica desarrollada por este autor, implementación de los algoritmos y revisión del artículo.

\section{REFERENCIAS}

[1] J. D. Garzón-Hidalgo; A. J. Saavedra-Montes, "Una metodología de diseño de micro redes para zonas no interconectadas de Colombia," TecnoLógicas, vol. 20, no. 39, pp. 39-53, May 2017. https://doi.org/10.22430/22565337.687

[2] D. López-García; A. Arango-Manrique; S. X. Carvajal-Quintero, "Integration of distributed energy resources in isolated microgrids: the Colombian paradigm,” TecnoLógicas, vol. 21, no. 42, pp. 13-30, May. 2018. https://doi.org/10.22430/22565337.774

[3] C.-H. Yoo; I. Y. Chung; H. J. Lee; S. S. Hong, "Intelligent Control of Battery Energy Storage for Multi-Agent Based Microgrid Energy Management," Energies, vol. 6, no. 10, pp. 4956-4979, Sep. 2013. https://doi.org/10.3390/en6104956

[4] J. M. Ramírez Scarpetta et al., "Control en Microrredes de A. C: Control Jerárquico, Tecnologías y Normativa,", Comité de Estudios C6 - Sistemas de distribución y generación dispersa, Documento técnico, Bogotá, 2020. URL

[5] M. N. Mojdehi; N. Webb, "Microgrid interoperability: First steps from policy to implementation," in 2016 IEEE Power and Energy Society Innovative Smart Grid Technologies Conference, ISGT, Minneapolis, 2016. https://doi.org/10.1109/ISGT.2016.7781025

[6] L. Che; M. Khodayar; M. Shahidehpour, "Only connect: Microgrids for distribution system restoration," IEEE Power Energy Mag., vol. 12, no. 1, pp. 70-81, Jan. 2014. https://doi.org/10.1109/MPE.2013.2286317

[7] A. Majzoobi; A. Khodaei, "Application of Microgrids in Supporting Distribution Grid Flexibility," IEEE Trans. Power Syst., vol. 32, no. 5, pp. 3660-3669, Sep. 2017. https://doi.org/10.1109/TPWRS.2016.2635024

[8] D. Wu; F. Tang; T. Dragicevic; J. C. Vasquez; J. M. Guerrero, "A Control Architecture to Coordinate Renewable Energy Sources and Energy Storage Systems in Islanded Microgrids," IEEE Trans. Smart Grid, vol. 6, no. 3, pp. 1156-1166, May. 2015. https://doi.org/10.1109/TSG.2014.2377018

[9] J. A. P. Lopes; C. L. Moreira; A. G. Madureira, "Defining Control Strategies for MicroGrids Islanded 
Operation," IEEE Trans. Power Syst., vol. 21, no. 2, pp. 916-924, May 2006. https://doi.org/10.1109/TPWRS.2006.873018

[10] H. Mahmood; D. Michaelson; J. Jiang, "Strategies for Independent Deployment and Autonomous Control of PV and Battery Units in Islanded Microgrids," IEEE J. Emerg. Sel. Top. Power Electron., vol. 3, no. 3, pp. 742-755, Sep. 2015. https://doi.org/10.1109/JESTPE.2015.2413756

[11] T. L. Vandoorn; J. C. Vasquez; J. De Kooning; J. M. Guerrero; L. Vandevelde, "Microgrids: Hierarchical Control and an Overview of the Control and Reserve Management Strategies," IEEE Ind. Electron. Mag., vol. 7, no. 4, pp. 42-55, Dec. 2013. https://doi.org/10.1109/MIE.2013.2279306

[12] J. Rocabert; A. Luna; F. Blaabjerg; P. Rodríguez, "Control of Power Converters in AC Microgrids," IEEE Trans. Power Electron., vol. 27, no. 11, pp. 4734-4749, Nov. 2012. https://doi.org/10.1109/TPEL.2012.2199334

[13] D. Wu; F. Tang; T. Dragicevic; J. C. Vasquez; J. M. Guerrero, "Autonomous Active Power Control for Islanded AC Microgrids With Photovoltaic Generation and Energy Storage System,” IEEE Trans. Energy Convers., vol. 29, no. 4, pp. 882-892, Dec. 2014. https://doi.org/10.1109/TEC.2014.2358612

[14] A. H. Fathima; K. Palanisamy, "Optimization in microgrids with hybrid energy systems - A review," Renew. Sustain. Energy Rev., vol. 45, pp. 431-446, May. 2015. https://doi.org/10.1016/J.RSER.2015.01.059

[15] G. Zhabelova; V. Vyatkin; V. N. Dubinin, "Toward Industrially Usable Agent Technology for Smart Grid Automation," IEEE Trans. Ind. Electron., vol. 62, no. 4, pp. 2629-2641, Apr. 2015. https://doi.org/10.1109/TIE.2014.2371777

[16] P. H. Nguyen; W. L. Kling; P. F. Ribeiro, "A Game Theory Strategy to Integrate Distributed Agent-Based Functions in Smart Grids," IEEE Trans. Smart Grid, vol. 4, no. 1, pp. 568-576, Mar. 2013. https://doi.org/10.1109/TSG.2012.2236657

[17] L. Hernandez et al., "A multi-agent system architecture for smart grid management and forecasting of energy demand in virtual power plants," IEEE Commun. Mag., vol. 51, no. 1, pp. 106-113, Jan. 2013. https://doi.org/10.1109/MCOM.2013.6400446

[18] C. P. Nguyen; A. J. Flueck, "Agent Based Restoration With Distributed Energy Storage Support in Smart Grids," IEEE Trans. Smart Grid, vol. 3, no. 2, pp. 1029-1038, Jun. 2012. https://doi.org/10.1109/TSG.2012.2186833

[19] B. Ramachandran; S. K. Srivastava; C. S. Edrington; D. A. Cartes, "An Intelligent Auction Scheme for Smart Grid Market Using a Hybrid Immune Algorithm,” IEEE Trans. Ind. Electron., vol. 58, no. 10, pp. 4603-4612, Oct. 2011. https://doi.org/10.1109/TIE.2010.2102319

[20] H. Dagdougui; R. Sacile, "Decentralized Control of the Power Flows in a Network of Smart Microgrids Modeled as a Team of Cooperative Agents," IEEE Trans. Control Syst. Technol., vol. 22, no. 2, pp. 510-519, Mar. 2014. https://doi.org/10.1109/TCST.2013.2261071

[21] C. M. Colson; M. H. Nehrir, "Comprehensive Real-Time Microgrid Power Management and Control With Distributed Agents," IEEE Trans. Smart Grid, vol. 4, no. 1, pp. 617-627, Mar. 2013. https://doi.org/10.1109/TSG.2012.2236368

[22] O. Palizban; K. Kauhaniemi; J. M. Guerrero, "Microgrids in active network management-Part I: Hierarchical control, energy storage, virtual power plants, and market participation," Renew. Sustain. Energy Rev., vol. 36, pp. 428-439, Aug. 2014. https://doi.org/10.1016/J.RSER.2014.01.016

[23] W. Liu; W. Gu; W. Sheng; X. Meng; Z. Wu; W. Chen, "Decentralized Multi-Agent System-Based Cooperative Frequency Control for Autonomous Microgrids With Communication Constraints," IEEE Trans. Sustain. Energy, vol. 5, no. 2, pp. 446-456, Apr. 2014. https://doi.org/10.1109/TSTE.2013.2293148

[24] Q. Li; F. Chen; M. Chen; J. M. Guerrero; D. Abbott, "Agent-Based Decentralized Control Method for Islanded Microgrids," IEEE Trans. Smart Grid, vol. 7, no. 2, pp. 637-649, Mar. 2016. https://doi.org/10.1109/TSG.2015.2422732

[25] C.-X. Dou; B. Liu, "Multi-Agent Based Hierarchical Hybrid Control for Smart Microgrid," IEEE Trans. Smart Grid, vol. 4, no. 2, pp. 771-778, Jan. 2013. https://doi.org/10.1109/TSG.2012.2230197

[26] N. L. Diaz; J. G. Guarnizo; M. Mellado; J. C. Vasquez; J. M. Guerrero, "A Robot-Soccer-Coordination Inspired Control Architecture Applied to Islanded Microgrids," IEEE Trans. Power Electron., vol. 32, no. 4, pp. 2728-2742, Apr. 2017. https://doi.org/10.1109/TPEL.2016.2572262

[27] A. Kantamneni; L. E. Brown; G. Parker; W. W. Weaver, "Survey of multi-agent systems for microgrid control," Eng. Appl. Artif. Intell., vol. 45, pp. 192-203, Oct. 2015. https://doi.org/10.1016/J.ENGAPPAI.2015.07.005

[28] M. Baun; M. A. Awadallah; B. Venkatesh, "Implementation of load-curve smoothing algorithm based on battery energy storage system," in 2016 IEEE Canadian Conference on Electrical and Computer Engineering (CCECE), Vancouver, 2016, pp. 1-5. https://doi.org/10.1109/CCECE.2016.7726668

[29] T. S. Mahmoud; D. Habibi; O. Bass, "Fuzzy logic for smart utilisation of Storage Devices in a typical microgrid," in 2012 International Conference on Renewable Energy Research and Applications (ICRERA), Nagasaki, 2012, pp. 1-6. https://doi.org/10.1109/ICRERA.2012.6477333 
[30] K. Alqunun; P. A. Crossley, "Rated energy impact of BESS on total operation cost in a microgrid," in 2016 IEEE Smart Energy Grid Engineering (SEGE), Oshawa, 2016, pp. $292-300$. https://doi.org/10.1109/SEGE.2016.7589540

[31] R. Morsali; S. Ghorbani; R. Kowalczyk; R. Unland, "On Battery Management Strategies in Multi-agent Microgrid Management," in Business Information Systems Workshops, Springer, Cham. 2017, pp. $191-202$. https://doi.org/10.1007/978-3-319-69023-0 17

[32] M. Batool; F. Shahnia; S. M. Islam, "Multi-level supervisory emergency control for operation of remote area microgrid clusters," J. Mod. Power Syst. Clean Energy, vol. 7, no. 5, pp. 1210-1228, Jan. 2019. https://doi.org/10.1007/s40565-018-0481-6

[33] F. Shahnia; S. Bourbour; A. Ghosh, "Coupling Neighboring Microgrids for Overload Management Based on Dynamic Multicriteria Decision-Making,” IEEE Trans. Smart Grid, vol. 8, no. 2, pp. 969-983, Mar. 2017. https://doi.org/10.1109/TSG.2015.2477845

[34] E. Bullich-Massagué; F. Díaz-González; M. Aragüés-Peñalba; F. Girbau-Llistuella; P. Olivella-Rosell; A. Sumper, "Microgrid clustering architectures," Appl. Energy, vol. 212, pp. 340-361, Feb. 2018. https://doi.org/10.1016/j.apenergy.2017.12.048

[35] M. Wooldridge, "Intelligent Agents: The Key Concepts," Multi-Agent Systems and Applications II, Springer, Berlin, Heidelberg, 2002, pp. 3-43. https://doi.org/10.1007/3-540-45982-0_1

[36] C. S. Karavas; G. Kyriakarakos; K. G. Arvanitis; G. Papadakis, "A multi-agent decentralized energy management system based on distributed intelligence for the design and control of autonomous polygeneration microgrids," Energy Convers. Manag., vol. 103, pp. 166-179, Oct. 2015. https://doi.org/10.1016/j.enconman.2015.06.021

[37] D. Montenegro; M. Hernandez; R. Dugan, OpenDSS-G (fomer DSSim-PC), 2013. URL

[38] F. Shahnia; S. Bourbour, "A practical and intelligent technique for coupling multiple neighboring microgrids at the synchronization stage," Sustain. Energy, Grids Networks, vol. 11, pp. 13-25, Sep. 2017. https://doi.org/10.1016/j.segan.2017.06.002

[39] J. A. Gil Tobón; M. A. Muñoz Marín, "Curva de Cargabilidad,” Derivado del curso de Instalaciones Eléctricas Industriales II, pp. 1-5, 2013. URL

[40] F. Z. Harmouch; N. Krami; N. Hmina, "A multiagent based decentralized energy management system for power exchange minimization in microgrid cluster," Sustain. Cities Soc., vol. 40, pp. 416-427, Jul. 2018. https://doi.org/10.1016/j.scs.2018.04.001

[41] IDEAM, “Atlas de Radiación Solar, Ultravioleta y Ozono de Colombia.” 2015. URL 